\title{
Metasurfaces for Reconfiguration of Multi-Polarization Antennas and Van Atta Reflector Arrays
}

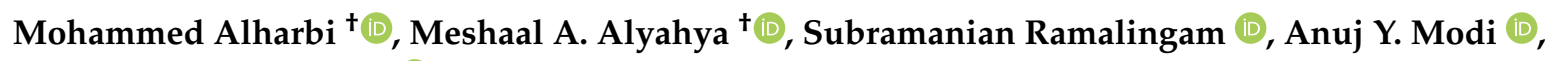 \\ Constantine A. Balanis * (1) and Craig R. Birtcher \\ School of Electrical, Computer, and Energy Engineering, Arizona State University, Tempe, AZ 85287, USA; \\ msalharb@asu.edu (M.A.); malyahy3@asu.edu (M.A.A.); subramanian.ramalingam@asu.edu (S.R.); \\ aymodi1@asu.edu (A.Y.M.); CRAIG.BIRTCHER@asu.edu (C.R.B.) \\ * Correspondence: balanis@asu.edu \\ + These authors contributed equally to this work.
}

Received: 9 July 2020; Accepted: 4 August 2020; Published: 6 August 2020

\begin{abstract}
This paper discusses the application of metasurfaces for three different classes of antennas: reconfiguration of surface-wave antenna arrays, realization of high-gain polarization-reconfigurable leaky-wave antennas (LWAs), and performance enhancement of van Atta retrodirective reflectors. The proposed surface-wave antenna is designed by embedding four square ring elements within a metasurface, which improves matching and enhances the gain when compared to conventional square-ring arrays. The design for linear polarization comprises of a $1 \times 4$ arrangement of ring elements, with a $0.56 \lambda$ spacing, placed amidst periodic patches. A $2 \times 2$ arrangement of ring elements is utilized for reconfiguration from linear to circular polarization, where a similar peak gain with better port isolation is realized. A prototype of the $2 \times 2$ array is fabricated and measured; a good agreement is observed between simulations and measurements. In addition, the concepts of the design of polarization-diverse holographic metasurface LWAs that form a pencil beam in the desired direction with a reconfigurable polarization are discussed. Moreover, recent developments incorporating polarization-reconfigurability in metasurface LWAs are briefly reviewed. In the end, the theory of van Atta arrays is outlined and their monostatic RCS is reviewed. A conventional retrodirective array is designed using aperture-coupled patch antennas with a microstrip-line feeding network, where the scattering from the structure itself degrades the performance of the reflector. This is followed by the integration of judiciously synthesized metasurfaces to reconfigure and improve the performance of retrodirective reflectarrays by removing the above-mentioned undesired scattering from the structure.
\end{abstract}

Keywords: reconfigurable antennas; surface wave array antenna; metasurface ground plane; low-profile; square-ring antennas; van Atta reflector; RCS reduction; structural mode scattering; metasurfaces; polarization reconfigurability; leaky waves

\section{Introduction}

Metasurfaces are two-dimensional, less bulky, and low-loss equivalents of metamaterials [1]. They have been popular as they are suitable for a variety of applications that include wavefront shaping and control [2,3], beamforming using high-gain leaky-wave antennas (LWAs) [4-6], radar cross section (RCS) reduction [7-9], and design of low-profile antennas [10,11]. These metasurfaces are realized as an array of patches on a grounded dielectric substrate, and they are easy to fabricate. By changing the geometry of these patches, their surface impedance and reflection phase can be controlled. A zero reflection phase can be realized at a particular frequency, thus mimicking a perfect magnetic conductor (PMC). Hence, they have been utilized to enhance the performance of antennas, 
in addition to the design of reconfigurable antennas. This paper details the utilization of metasurfaces to design and enhance the performance of three types of antennas: surface-wave antenna arrays, polarization-reconfigurable holographic LWAs, and van Atta retrodirective reflectors.

\subsection{Surface-Wave Antenna Arrays}

Proper excitation of surface waves enhances the overall antenna performance [12,13]. Consequently, several printed antennas have been reported, where the influence of surface waves has been examined. Monopole-like radiation has been achieved using a circular patch in the vicinity of uniform (square) and non-uniform metasurfaces [14,15]. Similarly, surface waves have been excited utilizing a square ring element surrounded by anisotropic metasurfaces, thus yielding broadside radiation [11]. Another design achieving broadside radiation has been realized by placing a diamond-shaped patch in the vicinity of square-patch metasurfaces [16].

To further improve the radiation performance, the analysis has been expanded to include multiple radiators in the vicinities of metasurfaces to form array metasurface antennas [16,17]. The reported designs require a specific spacing between the metasurface supercell to maintain and realize a high broadside gain. This spacing degrades the aperture efficiency and reduces the maximum attained gain. As it will be demonstrated in this paper, the spacings between the metasurface supercell can be reduced without disturbing the functionality of the design. The aforementioned designs are linearly polarized and have a wide range of potential low-profile applications. Circular polarization can be realized by placing a linear-to-circular metasurface superstrate above radiating elements [18]. This approach requires a certain spacing between the radiating element and the metasurface superstrate, which disturbs the overall height-profile. The second approach is to excite surface waves in two orthogonal planes that share the same magnitude and $90^{\circ}$ phase difference [19-21]. A truncated square patch element positioned in close proximity to square-patch metasurfaces resulted in $7 \mathrm{~dB}$ peak gain, a fractional bandwidth of $45.6 \%$, and 3-dB axial ratio (AR) bandwidth of $23.4 \%$ [19]. Another design implemented a planar slot to excite rectangular-patches metasurfaces and led to an average gain of $5.8 \mathrm{~dB}$, an impedance bandwidth of $33.7 \%$, and a $3-\mathrm{dB}$ AR bandwidth of $16.5 \%$ [20]. The low peak gain observed in the above-mentioned designs is improved to $12 \mathrm{~dB}$ by forming a $2 \times 2$ array of truncated patch elements positioned below square-patches metasurface [21]. This achieved gain can be further promoted by exciting surface waves along with the active element's fundamental mode, which will be demonstrated.

In the first part of this paper, which corresponds to surface-wave antenna arrays, an array of multiple square ring elements embedded within a metasurface is presented. With different array configurations, linear and circular polarizations are achieved.

\subsection{Polarization-Reconfigurable Holographic LWAs}

Metasurfaces have facilitated the realization of surface-impedance modulated periodic LWAs that are capable of forming fan or pencil beams with high gains and narrow beamwidths. One-dimensional LWAs form a fan beam in the desired angular direction, whereas two-dimensional LWAs form a pencil beam. The angular direction of the formed beam is determined by the modulation parameters of average surface reactance and period of modulation. One-dimensional periodic metasurface LWAs were realized in [22], while two-dimensional LWAs were designed using the principle of holography in [4].

The polarization of the formed beam is dependent on the polarization of the leaky-wave mode; TM-mode leaky waves result in vertically-polarized beams, while TE-mode leads to horizontally polarized [23]. Circularly-polarized beams were realized by employing a spiral surface impedance modulation in [24]. The design of scalar holographic metasurfaces to form a pencil beam in the desired direction with a desired polarization was detailed in [25]. Tensor impedance surfaces with amplitude, phase, and polarization control were proposed in [26]. 
The suitability of these metasurfaces to communication-based applications necessitates the formation of beams with reconfigurable polarizations. The design of polarization-diverse metasurfaces that can form a pencil beam in the desired direction with a horizontal, vertical, or circular polarization is discussed in [6]. The polarization state was reconfigured by changing the source of excitation. A similar approach was used to realize dual reconfigurable polarizations using tensor impedance surfaces, and recently proposed in [27]. Another recently-proposed design achieves polarization reconfigurability by using polarization-insensitive holographic surfaces, where the reconfiguration is facilitated by diodes on the surface-wave launchers [28].

The second part of this paper reviews the procedures to design a multi-polarization metasurface LWA. The existing techniques are reviewed and potential new techniques are proposed.

\subsection{Van Atta Retrodirective Reflectors}

In many radar and communication systems, reflectors are utilized because of their ability to maximize the re-radiation towards the direction of wave incidence. Such a response can be achieved using a retrodirective reflector, first proposed in [29]. The retrodirective reflector is an antenna array whose elements are interconnected by transmission lines such that the received signal is then reradiated towards the direction of incidence [30-32]. In automotive collision avoidance systems, a high scattered field can be achieved only toward near-normal directions to the target surface. Thus, by equipping vehicles and road obstacles with retrodirective reflectors, the self-phasing feature of such reflectors will increase scattering beamwidths and targets will become more visible [32]. Furthermore, retrodirective reflectors have been investigated for the application of wireless power transfer [33-35]. A device that requires wireless power can send a beacon signal that is then received, amplified, and sent back to the user by the retrodirective antenna array.

Generally, retrodirective arrays can be synthesized with basic radiating elements such as dipoles [31]. In addition, they can be designed using patch antennas [36-38]. However, due to the high-backscattering from the structure (consisting of antenna arrays), it degrades the performance of the retrodirective reflector by destructively interfering with the desired reradiated fields. Thus, a low-backscattering array of long slots was investigated [39], and it was shown that this reflector could reradiate fields without interference from the array structure's scattering. However, a retrodirective reflector comprised of a patch antenna array is a better option due to the simplicity of design and the low profile of patch antenna elements if the high backscattering by the patch antenna array can be mitigated. Thus, artificial magnetic conductor (AMC) technology is utilized to reconfigure the performance of such antenna arrays by reducing scattering from such structures [40].

In the third part of this paper, a van Atta retrodirective reflector with a smoother backscattering pattern is synthesized and developed using a two-dimensional microstrip-antenna array. Conventional retrodirective reflectors are sensitive to the interference by the fields scattering from the antenna structure. Using a virtual feeding network, structural mode scattering is identified and canceled using metasurfaces.

\section{Surface-Wave Antenna Arrays}

This section details the first of the three areas, where metasurfaces are used to realize and enhance the antenna performance. In this section, a $1 \times 4$ arrangement of square ring elements is printed on the same plane with periodic patches to achieve linear polarization. With a spacing of $0.56 \lambda_{o}$ between the elements, a high realized gain with high aperture efficiency is attained. The polarization can be reconfigured from linear to circular using a $2 \times 2$ structure with a spacing of $0.85 \lambda_{0}$ between the ring elements. After analyzing the port isolations between the ports, a simple parallel feeding network is designed and incorporated with the structures. Due to the similar characteristics of the two arrays, only the $2 \times 2$ circular polarized structure is experimentally validated. 


\section{1. $1 \times 4$ Linearly Polarized Metasurface Array}

The array designs considered in this paper are based on the structure proposed in [11]. The radiator within the metasurface (RWMS) is extended due to its wide matching bandwidth and stable unidirectional radiation. The mechanism of this design depends on the excitation of the radiating elements' mode (TM11) and the surface-wave modes in such a way that their resonances are closely spaced in order to enhance the overall antenna performance in terms of gain and bandwidth. For brevity, this paper does not cover the analysis and the design of RWMS; the readers can refer to [11] to develop an understanding of the RWMS design. A one-dimensional (1-D) array is designed, as illustrated in Figure 1a. The array has four square-ring elements utilized to launch surface waves, in addition to their resonances. The inter-element spacing is $s=37.5 \mathrm{~mm}$, which is about $0.56 \lambda_{o}$ at $4.5 \mathrm{GHz}$. The substrate is Rogers RT/Duroid-5880 $\left(\varepsilon_{r}=2.2, \tan \delta=0.0009\right)$ with a thickness $h=5.08 \mathrm{~mm}$. The ring elements and the metasurface patches are printed on the top face of a single substrate with length $l_{s}=191.5 \mathrm{~mm}$ and width $w_{s}=62 \mathrm{~mm}$. The remaining parameters are detailed in the caption of Figure 1. Since the design relies on the excitation of surface waves, it would be of interest to shed insight on the isolation between the ports. Therefore, the ring elements are individually fed with four coaxial probes, as presented in Figure 1a. For optimum broadside radiation, all elements are excited with the same magnitude and phase.

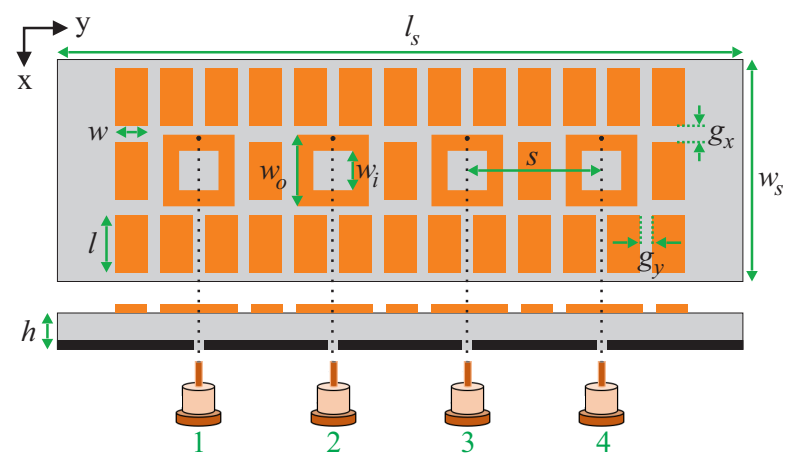

(a) RWMS with coaxial ports

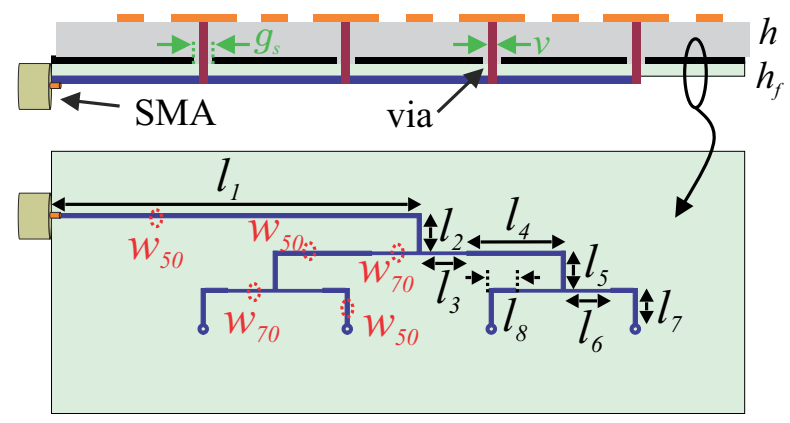

(b) RWMS with feed network

Figure 1. The $1 \times 4$ square ring elements embedded within metasurface and excited with (a) four coaxial probes and (b) feed network. The RWMS parameters are: $w_{i}=10 \mathrm{~mm}, w_{0}=20 \mathrm{~mm}, l=16 \mathrm{~mm}$, $w=9 \mathrm{~mm}, g_{x}=4.5 \mathrm{~mm}$, and $g_{y}=3.5 \mathrm{~mm}$. The feed network parameters are: $l_{1}=96.3 \mathrm{~mm}, l_{2}=10 \mathrm{~mm}$, $l_{3}=11.85 \mathrm{~mm}, l_{4}=25.65 \mathrm{~mm}, l_{5}=10 \mathrm{~mm}, l_{6}=11.85 \mathrm{~mm}, l_{7}=9.29 \mathrm{~mm}, l_{8}=6.9 \mathrm{~mm}, g_{s}=2.74 \mathrm{~mm}$, $v=1.143 \mathrm{~mm}, w_{50}=1.1 \mathrm{~mm}$, and $w_{70}=0.7 \mathrm{~mm}$.

The reflection coefficients and the mutual coupling between the coaxial ports are simulated and presented in Figure $2 \mathrm{a}$. The $-10 \mathrm{~dB}$ reflection coefficient bandwidth for all ports (dotted red line) is about $28 \%$ from $3.9-5.2 \mathrm{GHz}$, which is the same as attained with a single RWMS [11]. The isolation between two adjacent elements (e.g., $S_{12}$ ) is low at lower frequencies and reaches up to $-10 \mathrm{~dB}$ at 5.2 GHz. This is attributed to surface waves along the y-axis, which begin to resonate above $5 \mathrm{GHz}$ [11], 
and they disturb both the port isolation and the broadside radiation. The isolation can be improved by increasing the spacing between the square-ring elements; however, this approach will introduce undesired sidelobes.

Once the port isolations are examined, a parallel feeding network, presented in Figure 1b, is designed for the implementation of the RWMS array. The feeding network is also designed on Rogers RT/Duroid-5880 substrate whose thickness $h_{f}=0.38 \mathrm{~mm}$. The feed is stacked below the $1 \times 4$ array, and the outputs of the feed are coupled to the ring elements by metallic vias. The dimensions of the feed network are detailed in the caption of the figure. For comparison purposes, the S-parameters investigated for the feed network when it is connected to the RWMS and a conventional square element array (in the absence of metasurface). As illustrated in Figure 2a, when the feed is connected to the RWMS array, the $-10 \mathrm{~dB}$ fractional bandwidth is comparable to that obtained with coaxial ports; however, poor matching is attained when the feed is integrated with a conventional $1 \times 4$ square ring array.

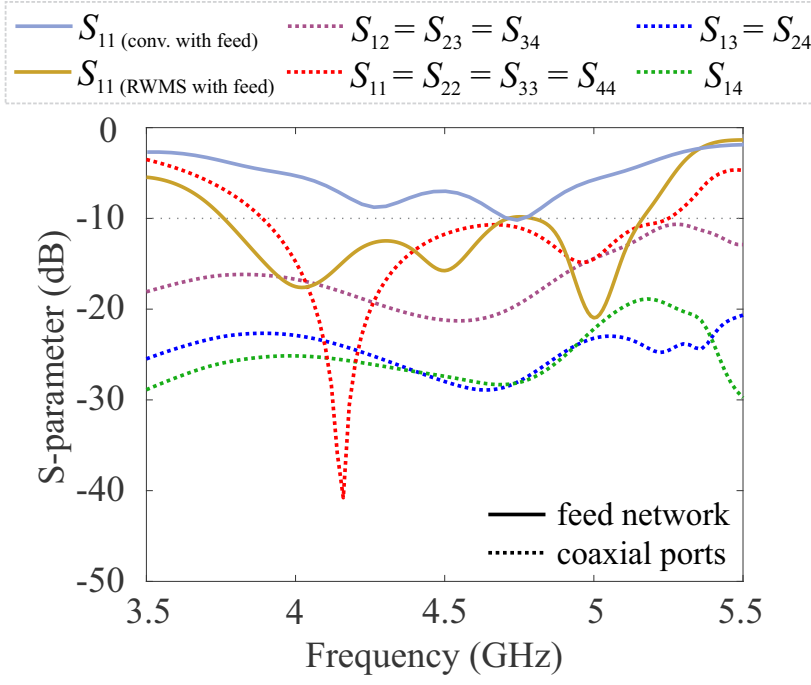

(a)

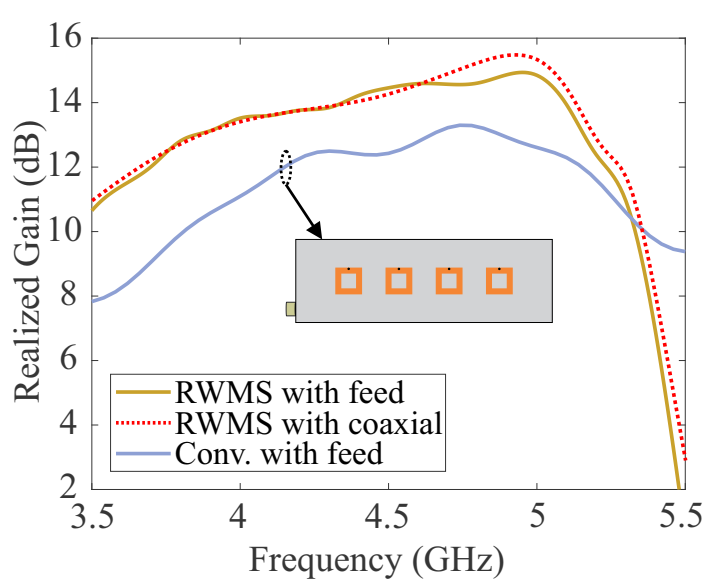

(b)

Figure 2. (a) S-parameters and (b) the broadside realized gain of the $1 \times 4$ RWMS array.

To further examine the influence of coupling on the radiation behavior, the broadside realized gain is examined and plotted in Figure $2 b$. At lower frequencies, the gain is the same when the RWMS is excited either by the feed network or the multiple coaxial probes; however, the two results begin to deviate slightly around $4.7 \mathrm{GHz}$, which is attributed to the more pronounced effect of coupling.

The simulated realized gain, when the RWMS is excited with a feed network, ranges from $13.5 \mathrm{~dB}$ at $4 \mathrm{GHz}$ and reaches up to $14.8 \mathrm{~dB}$ at $5 \mathrm{GHz}$. This observed high gain for the RWMS is attributed to the use of metasurfaces, as displayed in Figure $2 \mathrm{~b}$. The aperture efficiency at $4.5 \mathrm{GHz}$ is about $83 \%$ for the RWMS array, while it is only $52 \%$ for a conventional square ring array. The impact of the metasurface in enhancing the efficiency is demonstrated by plotting the surface current distribution at $4.5 \mathrm{GHz}$ in Figure 3. As observed, both the ring elements and the periodic patches contribute to the total radiation; this justifies the higher achieved efficiency compared to the conventional array. 


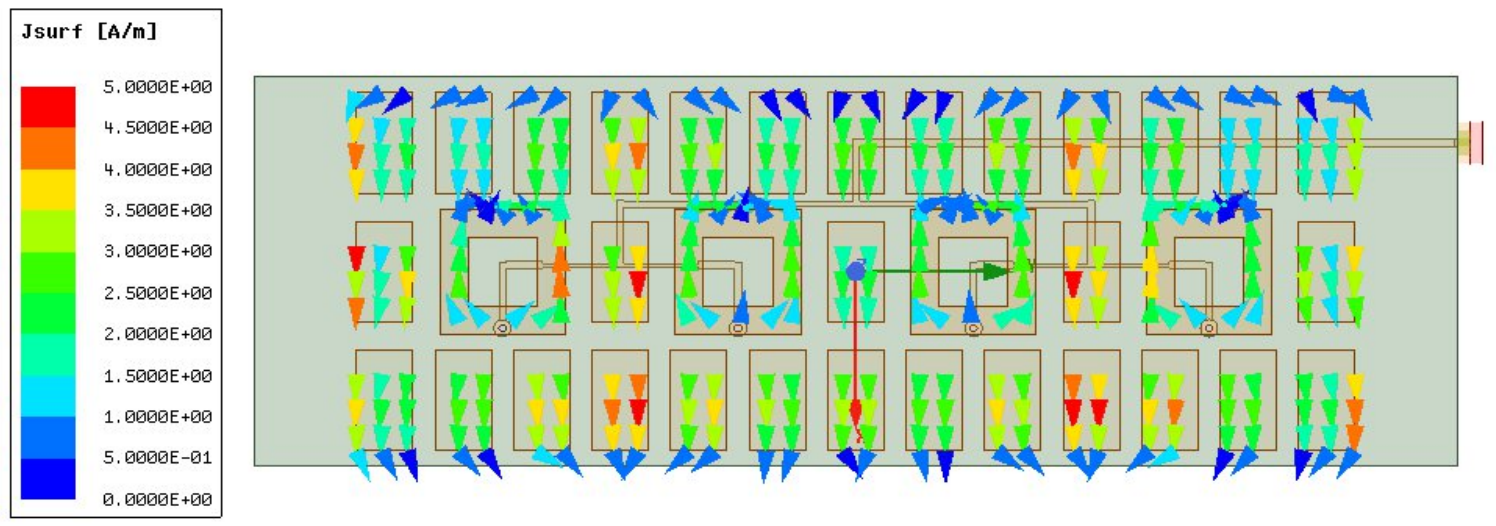

Figure 3. Surface current distribution at $4.5 \mathrm{GHz}$ for the $2 \times 2$ metasurface array.

\section{2. $2 \times 2$ Circularly Polarized Metasurface Array}

This section addresses the reconfiguration from the linear to circularly polarized RWMS array. Since RWMS is designed to excite surface waves on a single plane [11], circular polarization (CP) can be achieved by arranging orthogonal structures of RWMS fed with a 90-degree phase difference. To achieve a gain comparable to the linear RWMS, a $2 \times 2$ RWMS scheme is considered and designed, as displayed in Figure 4a.

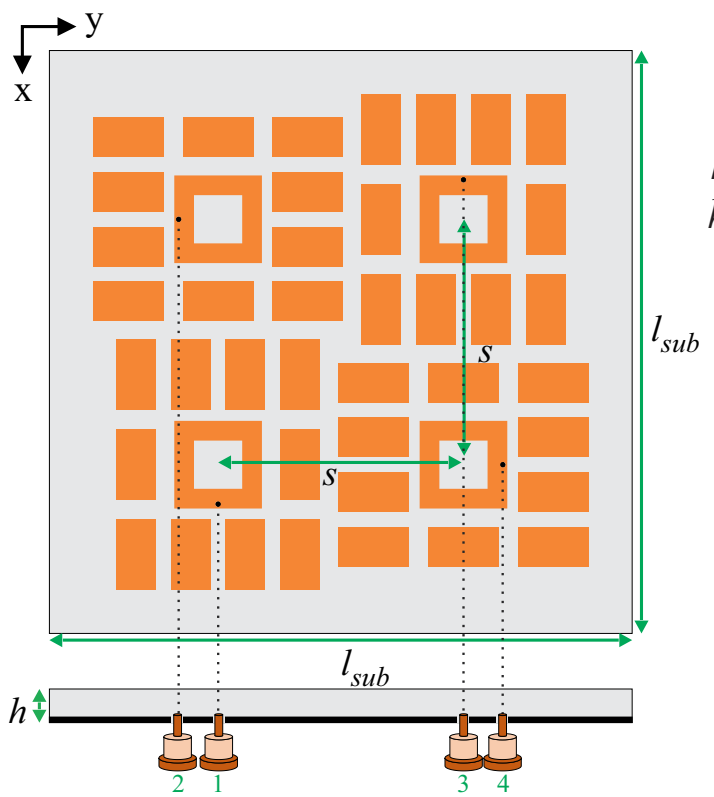

(a) Structure is fed with four coaxial ports

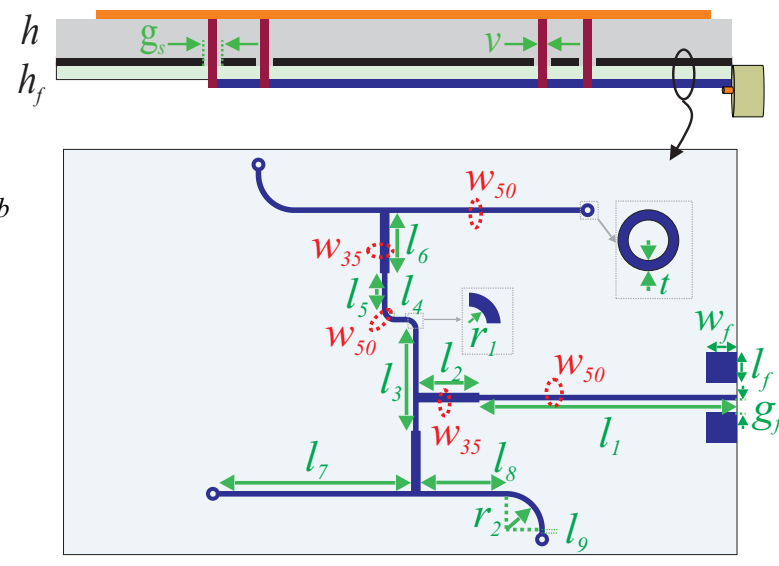

(b) Structure is fed with feed network

Figure $4.2 \times 2$ square ring elements embedded within metasurface and excited with (a) four coaxial probes, and (b) feed network. The feed network parameters are: $l_{1}=50.875 \mathrm{~mm}, l_{2}=12 \mathrm{~mm}, l_{3}=20 \mathrm{~mm}$, $l_{4}=2.25 \mathrm{~mm}, l_{5}=7.2 \mathrm{~mm}, l_{6}=11.9 \mathrm{~mm}, l_{7}=37.87 \mathrm{~mm}, l_{8}=16.92 \mathrm{~mm}, l_{9}=0.69 \mathrm{~mm}, w_{50}=1.1 \mathrm{~mm}$, $w_{35}=1.9 \mathrm{~mm}, g_{s}=2.74 \mathrm{~mm}, v=1.143 \mathrm{~mm}, w_{f}=l_{f}=6 \mathrm{~mm}, g_{f}=2.45 \mathrm{~mm}, r_{1}=1.4 \mathrm{~mm}, r_{2}=6.5 \mathrm{~mm}$, and $t=0.79 \mathrm{~mm}$.

A square grounded dielectric is modeled with a thickness $h=5.08 \mathrm{~mm}$, and a length $l_{\text {sub }}=133 \mathrm{~mm}$. The spacing $s$ along the $x$ - and $y$-planes is $56 \mathrm{~mm}$, which corresponds to $0.85 \lambda_{o}$ at 4.5 GHz. The dielectric material, the dimensions of the ring elements, and the metasurface patches are identical to those given in the $1 \times 4$ array configurations. Similar to the linear array, the coupling between the ring elements is investigated first by individually feeding the ring elements with four coaxial probes. The simulated scattering parameters for $2 \times 2$ circular polarized structure are depicted 
in Figure 5a. The $-10 \mathrm{~dB}$ fractional bandwidth is slightly less than that for the $1 \times 4$ array, with a bandwidth of $25 \%$ from 3.9 to $5.0 \mathrm{GHz}$. The mutual coupling between vertically/horizontally adjacent ports and diagonally orientated ports is less than $-20 \mathrm{~dB}$, which is lower than those observed for the $1 \times 4$ linear array. This is attributed to the orthogonality of the excited surface waves, which agrees with the findings in [41].

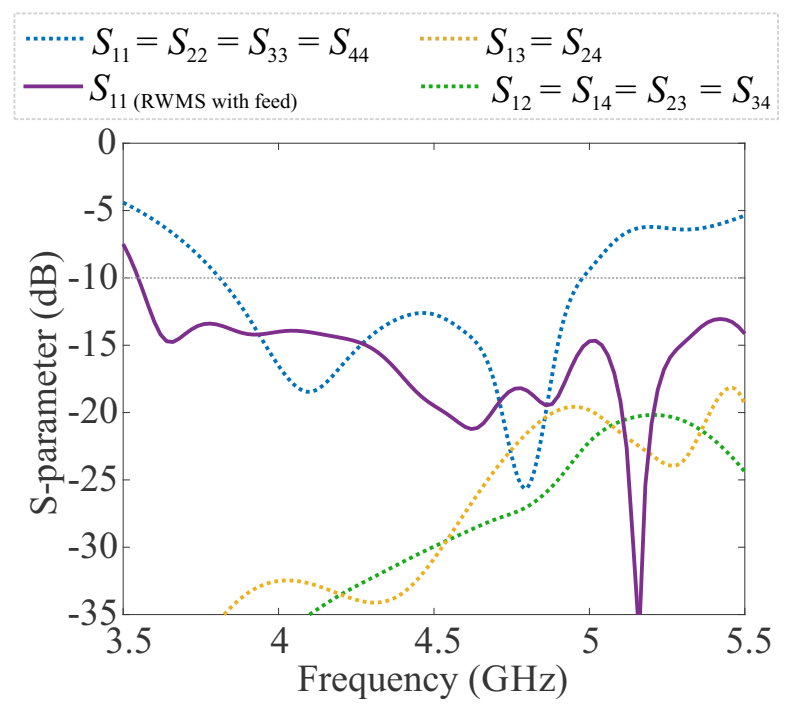

(a)

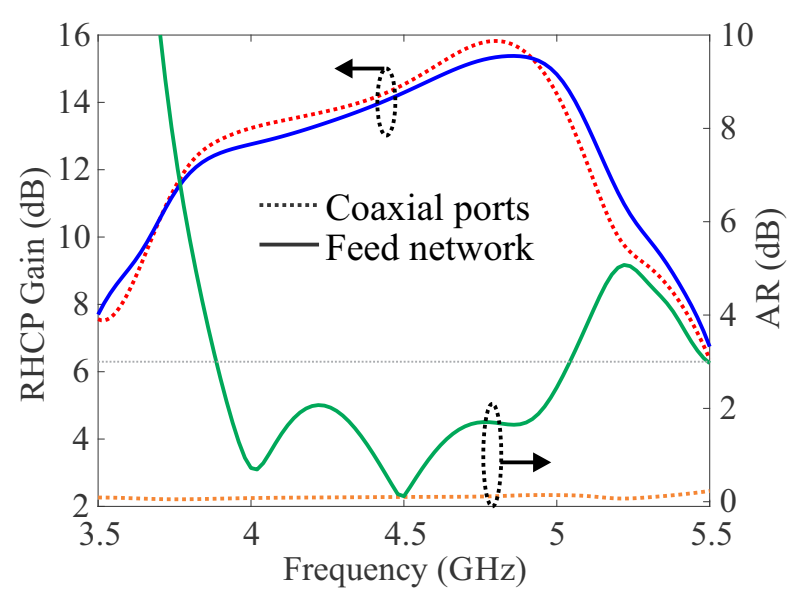

(b)

Figure 5. (a) S-parameters and (b) the broadside RHCP gain along with the axial ratio of the $2 \times 2$ RWMS scheme.

Once the coupling is examined, a parallel feeding network is designed to excite all four ring elements with a single coaxial feed, as illustrated in Figure $4 \mathrm{~b}$. The feed is designed at the center frequency $(4.5 \mathrm{GHz})$ to split the power equally among the four ring elements with a $90^{\circ}$ phase increment. The feed can dictate whether the sense of rotation of the CP is right hand (RHCP) or left hand (LHCP), depending on the orientation of the phase increment (clockwise or counterclockwise); in this work, the feed is modeled to realize RHCP. The feed is designed on Rogers RT/Duroid-5880 substrate, which has a thickness $h_{f}=0.38 \mathrm{~mm}$. Similar to the $1 \times 4$ linear array, the feeding substrate is stacked below the $2 \times 2$ circular array, and the outputs of the feed network are coupled to the ring elements using vias. The remaining dimensional parameters of the feeding network are detailed in the caption of Figure 4 . When the feed network excites the $2 \times 2$ RWMS, the $-10 \mathrm{~dB}$ matching bandwidth is larger than that obtained with the four coaxial feeds. This is attributed to the parallel feed structures 
which do not provide any isolation between the ports; therefore, any reflected power from any port can couple through to other ports resulting in small power return back to the feed.

To further analyze the performance of the $2 \times 2$ RWMS structure, the broadside realized gain and the axial ratio are examined. The simulated results for the two different feed approaches are presented in Figure $5 b$.

When the structure is fed with individual probes, the realized gain is $13.5 \mathrm{~dB}$ at $4 \mathrm{GHz}$ and reaches up to $15.8 \mathrm{~dB}$ at $4.8 \mathrm{GHz}$. Almost similar gain is attained when the feed network is utilized. However, there is a noticeable discrepancy in the axial ratio between the two feeding methods. For the individual feed approach, the broadside axial ratio (AR) is below $0.25 \mathrm{~dB}$ over the design frequency range, while it is only below $3 \mathrm{~dB}$ over a more narrow frequency range $(3.9 \mathrm{GHz}$ to $5.1 \mathrm{GHz})$ when the structure is excited with the feed network. The fluctuation in the axial ratio magnitude is due to the poor isolation of the considered feeding network, which leads to magnitude and phase errors for the power reached to each port [42].

The broadside realized gain at the center frequency $(4.5 \mathrm{GHz})$ is around $14.5 \mathrm{~dB}$, while it is only $10 \mathrm{~dB}$ for a $2 \times 2$ conventional array. This corresponds to the aperture efficiencies of $56 \%$ and $20 \%$ for the metasurface array and its conventional counterpart, respectively. Similar to the linear metasurface array, the high attained efficiency for the $2 \times 2$ metasurface array is due to the excitation of the radiating element's mode along with the surface waves' modes. This can be verified by examining the surface current distribution in Figure 6, which shows that both the radiating elements and the periodic patches contribute to the total radiation.

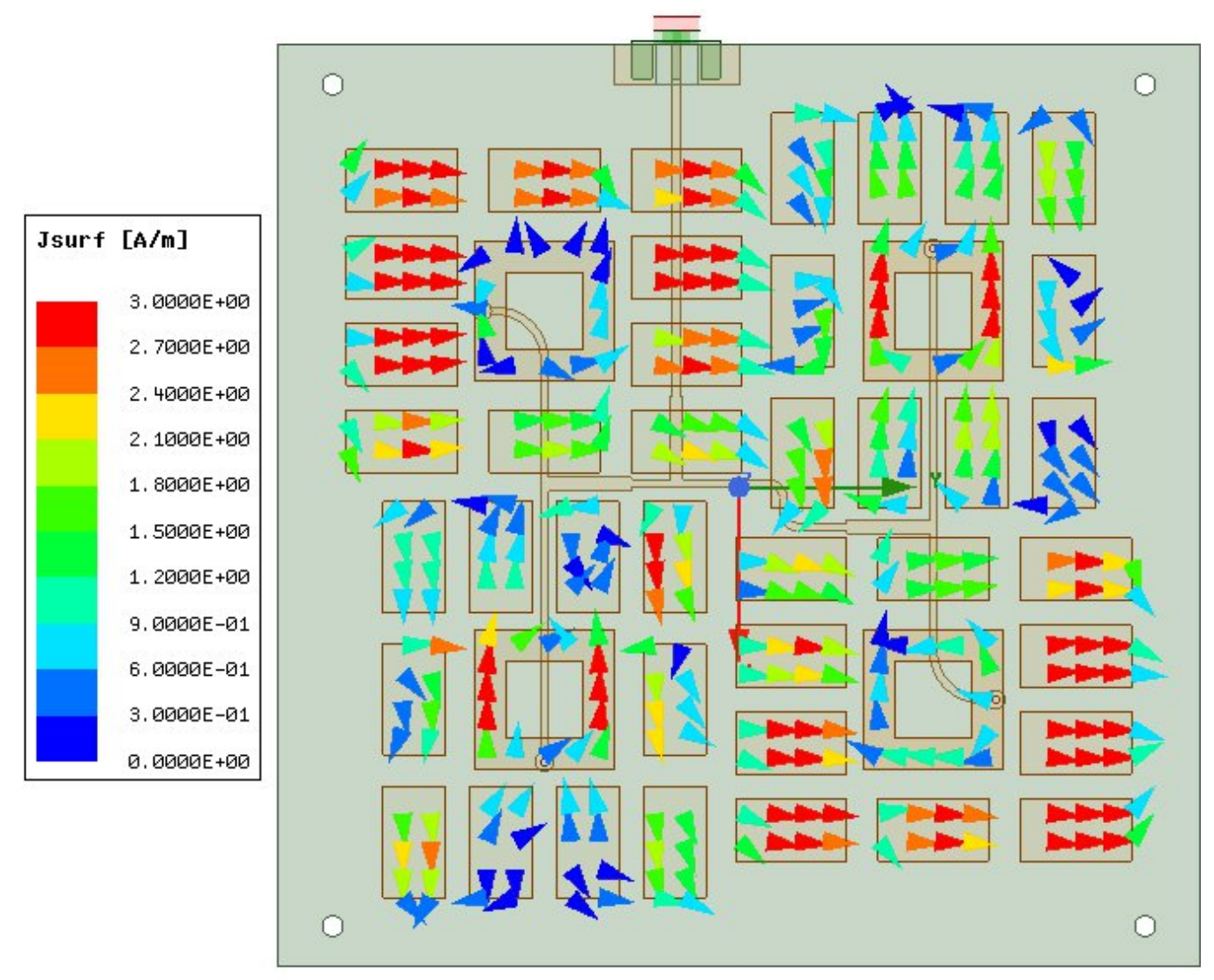

Figure 6. Surface current distribution at $4.5 \mathrm{GHz}$ for the $2 \times 2$ metasurface array.

\subsection{Fabrication and Measurements}

To experimentally validate the simulated data, the proposed $2 \times 2$ RWMS circular array was fabricated, as depicted in Figure 7. 

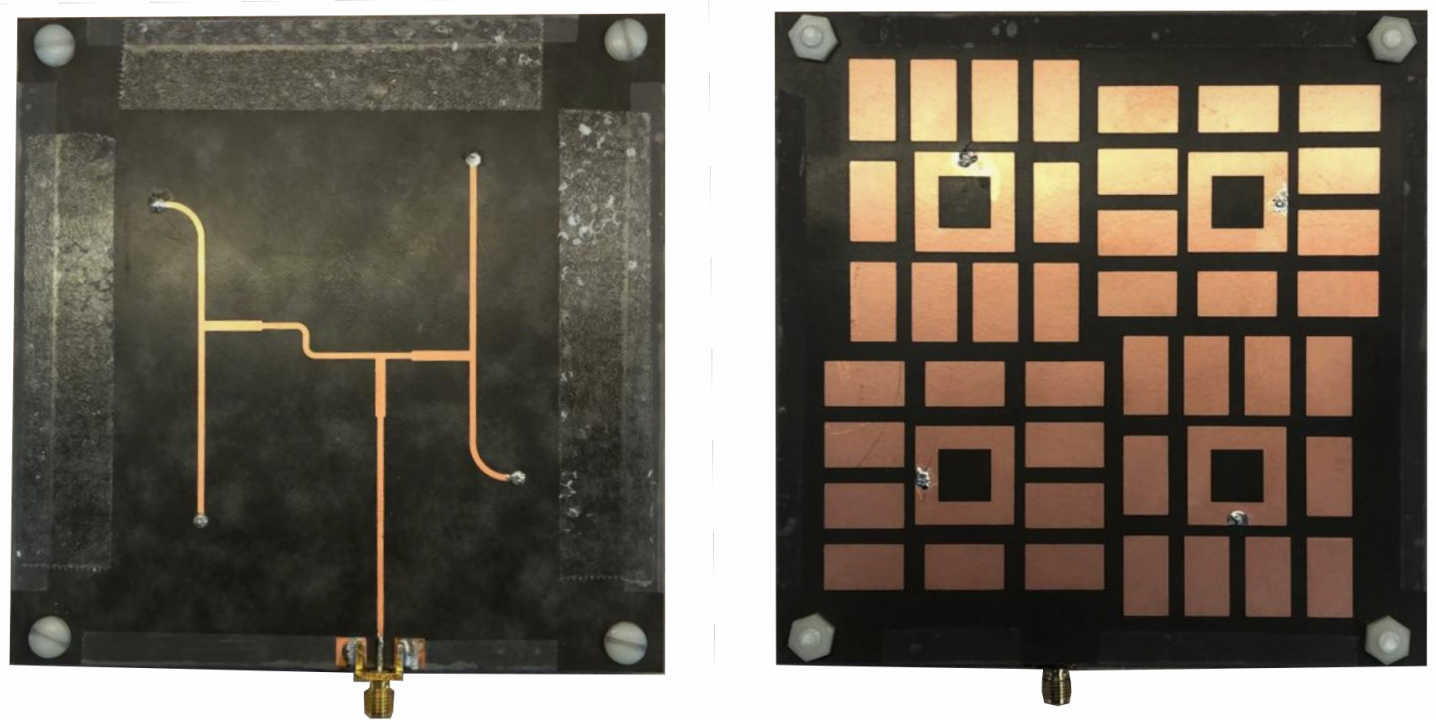

Figure 7. Top and bottom views of the fabricated prototype of the $2 \times 2$ RWMS array incorporated with the feed network.

Since linear and circular RWMS arrays share similar characteristics, only the circular array was considered for fabrication. The substrate materials and the dimensions of the fabricated design were identical to those specified in Figure 4. An edge mount SMA connector was soldered to the feed network substrate, as demonstrated in Figure 7. Four nylon screws were placed at the corner to tighten the feed and RWMS substrates together and avoid any misalignment.

The comparison of the measured and simulated results are displayed in Figures 8 and 9. The simulated and measured $S_{11}$ and the axial ratio showed a good agreement; however, the broadside RHCP gain was about $1 \mathrm{~dB}$ lower than the simulated data. This deviation could be attributed to the fabrication tolerance and the stacking assembly error. The measured and simulated radiation patterns along different planes are displayed in Figure 9; a good agreement was observed between the simulated and measured data.

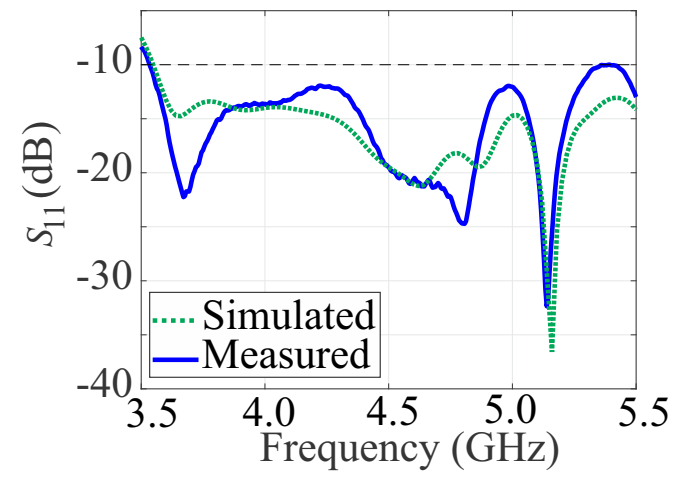

(a)

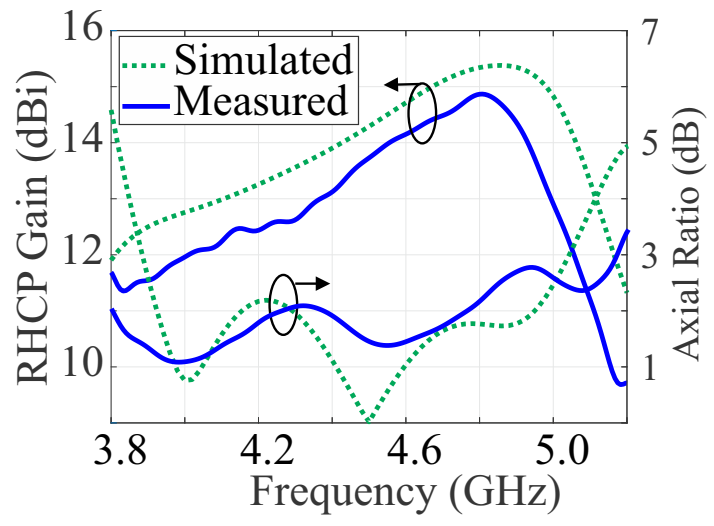

(b)

Figure 8. Comparison between simulated and measured values of the (a) reflection coefficient, (b) RHCP broadside gain, and axial ratio. 


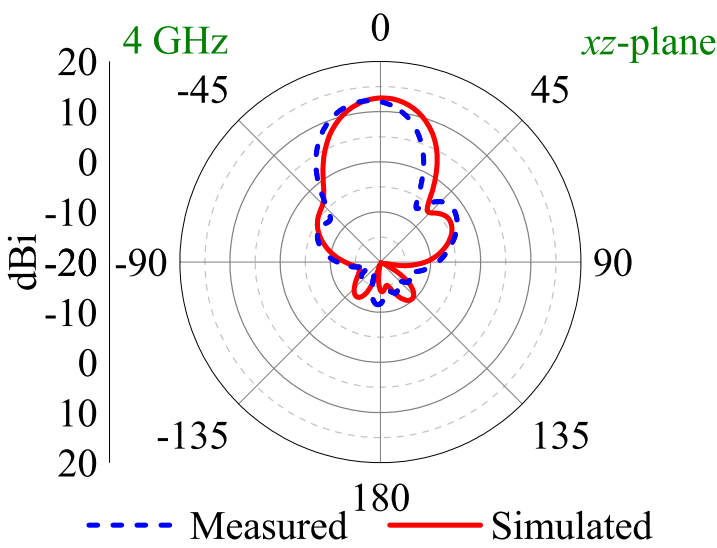

(a)

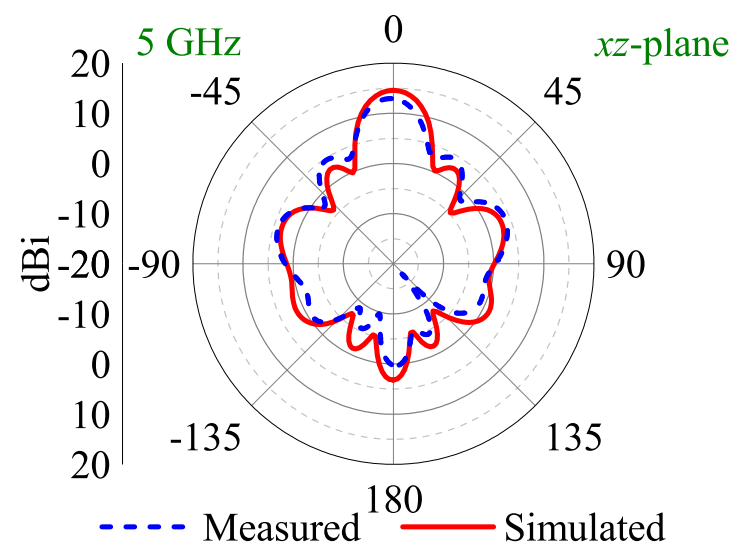

(c)

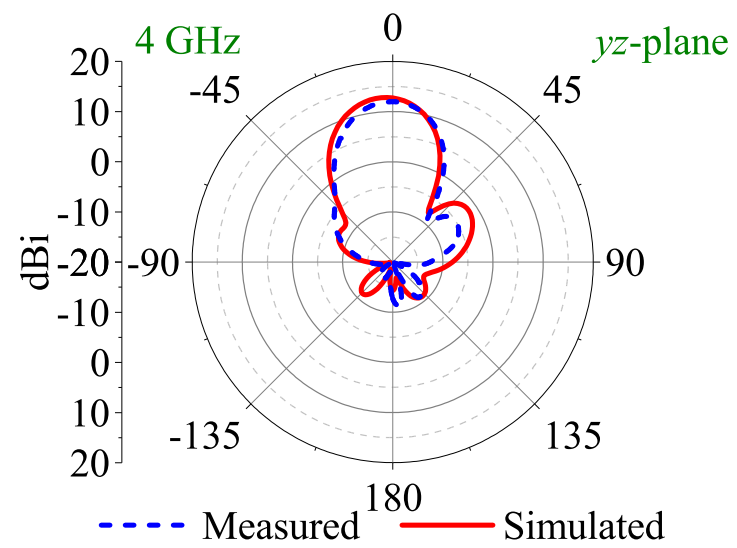

(b)

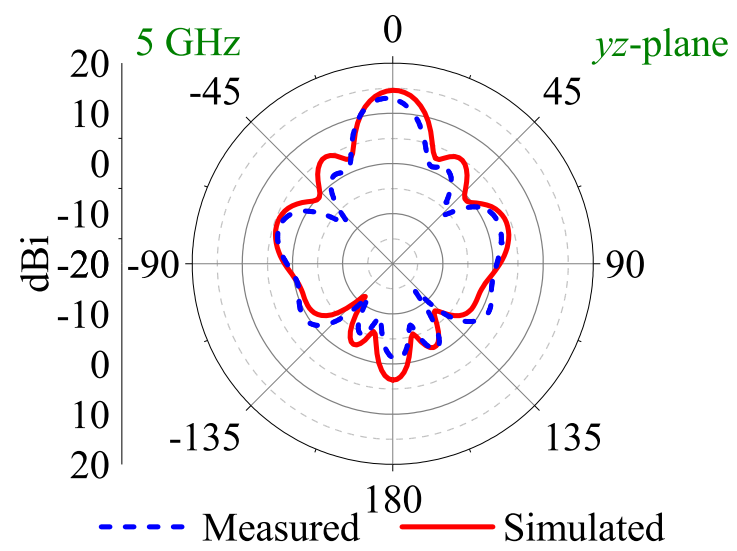

(d)

Figure 9. Simulated and measured radiation patterns at (a) $4 \mathrm{GHz}$ along $x z$-plane, (b) $4 \mathrm{GHz}$ along yz-plane, (c) $5 \mathrm{GHz}$ along $x z$-plane and (d) $5 \mathrm{GHz}$ along yz-plane.

\section{Polarization Reconfigurable Holographic LWAs}

The advent of metasurfaces has facilitated the realization of surface-reactance modulation by varying the geometries of patches that are periodically arranged on a dielectric-covered ground plane. Patches with an inductive reactance support TM-mode surface waves while capacitive surface reactance support TE-mode [43]. The surface reactance of a metasurface unit cell depends on the frequency of operation and can be determined using the transverse-resonance method (TRM) [23].

The surface-reactance modulation function $X_{S}$ required to form a pencil beam, in the desired elevation angle $\theta_{d}$ (from broadside) and the azimuth angle $\phi_{d}$, is obtained using the holographic principle $[4,23]$

$$
X_{s}(x, y)=X_{a v g}\left\{1+M e^{j \beta \sqrt{\left(x-x_{c}\right)^{2}+\left(y-y_{c}\right)^{2}}} e^{j k_{0}\left(x \sin \theta_{d} \cos \phi_{d}+y \sin \theta_{d} \sin \phi_{d}\right)}\right\}
$$

where $X_{a v g}$ is the average surface reactance, $M$ is the modulation index, $\left(x_{c}, y_{c}\right)$ is the location of the source and $k_{0}$ is the free-space phase constant. This leads to a holographic pattern which is a set of periodic ellipses that have a focus at the location of the source, as shown in Figure 10.

The metasurface forms a pencil beam with a linear polarization depending on the leaky-wave mode. The pencil beam is formed as a superposition of radiation from forward and backward leaky waves that move in opposite directions with a change in frequency. Hence, they have to be operated at the phase-crossover frequency, where the radiation from the forward and backward leaky waves interfere constructively. 


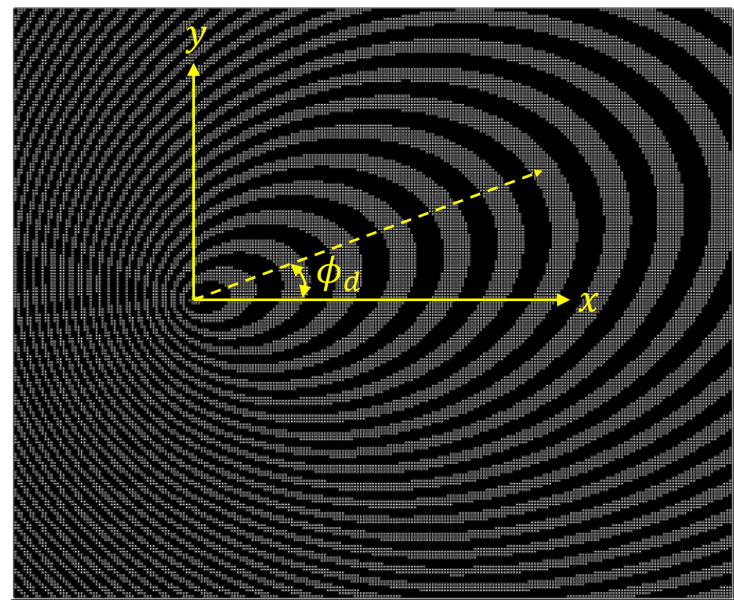

Figure 10. The holographic pattern of the surface-reactance profile needed to form a pencil beam along $\left(\theta_{d}, \phi_{d}\right)$.

Different polarizations can be obtained by performing geometric modifications along the surface [25]. While operating at non-phase crossover frequencies, vertical and horizontal polarizations can be achieved by including a $180^{\circ}$ phase shift on either side of the principal axes of the ellipses; a $180^{\circ}$ phase shift along the minor axis results in vertical polarization, while that along the major axis leads to horizontal. A phase shift of $180^{\circ}$ on either side of both the major and minor axes, along with a relative phase of $90^{\circ}$ between them, leads to a circularly polarized pencil beam. The principal axes of the ellipses are expressed as

$$
\begin{aligned}
& \left(x-x_{c}\right) \cos \phi_{d}+\left(y-y_{c}\right) \sin \phi_{d}=0 \\
& \left(x-x_{c}\right) \sin \phi_{d}-\left(y-y_{c}\right) \cos \phi_{d}=0
\end{aligned}
$$

The patterns pertaining to horizontal, vertical, and circular polarization are shown in Figure 11.

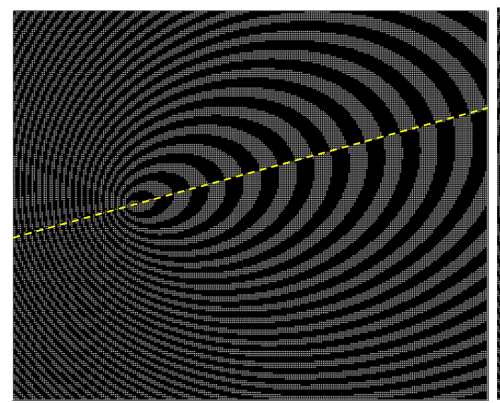

(a)

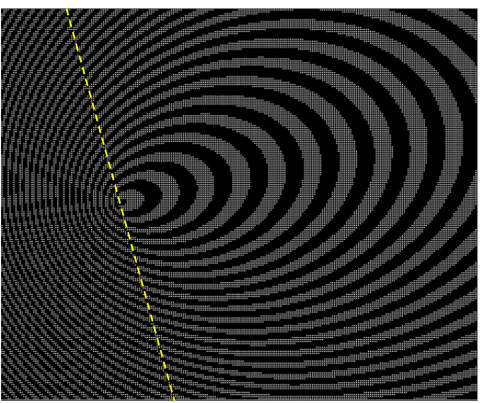

(b)

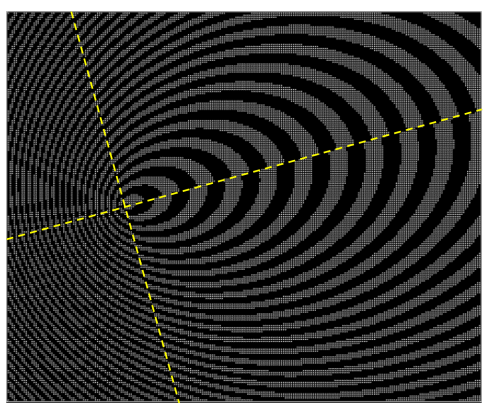

(c)

Figure 11. The holographic pattern of the surface-reactance profile needed to form a pencil beam with (a) horizontal, (b) vertical, and (c) circular polarizations.

Polarization reconfigurability can be achieved by feeding the metasurface with two sources instead of one [6]. The surface-reactance modulation function can be obtained as a superposition of the surface-reactance profile needed for each polarization that includes a $90^{\circ}$ phase shift between them. Vertically- and horizontally-polarized beams can be formed when the metasurface is excited by the individual sources, and a circularly polarized beam is formed when excited by both sources, as illustrated in Figure 12.

This way, a single metasurface is capable of forming a pencil beam in the desired direction with a horizontal, vertical, or circular polarization. The E-plane radiation patterns demonstrating the polarization reconfigurability is shown in Figure 13. 


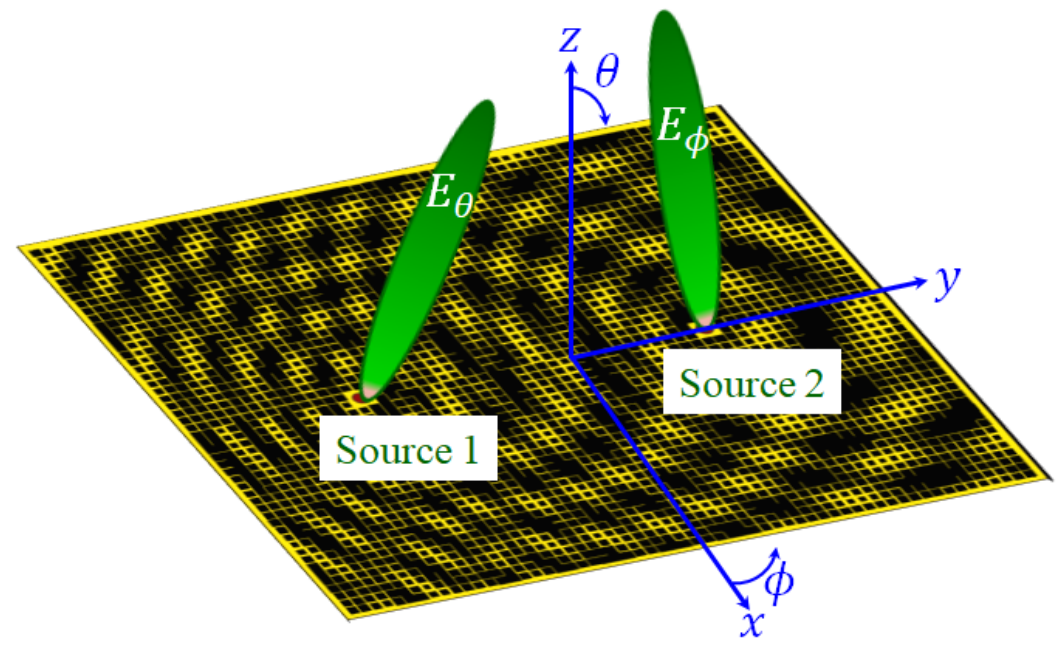

Figure 12. A holographic metasurface, fed by two sources, that forms a pencil beam with three reconfigurable polarization states: vertical, horizontal, and circular polarizations [23].

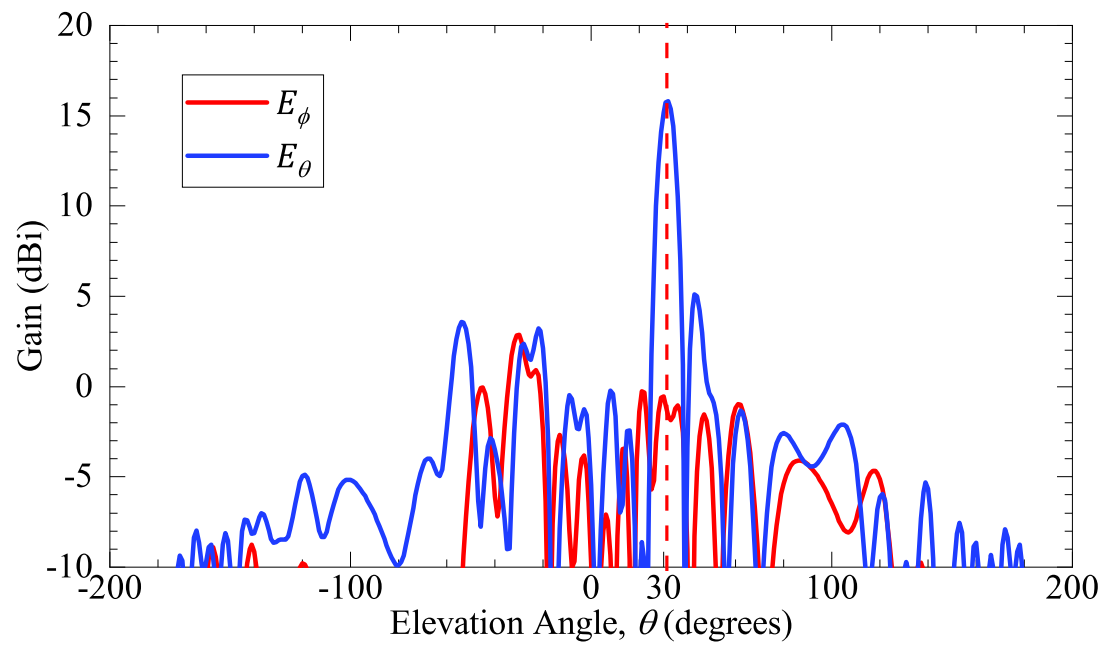

(a)

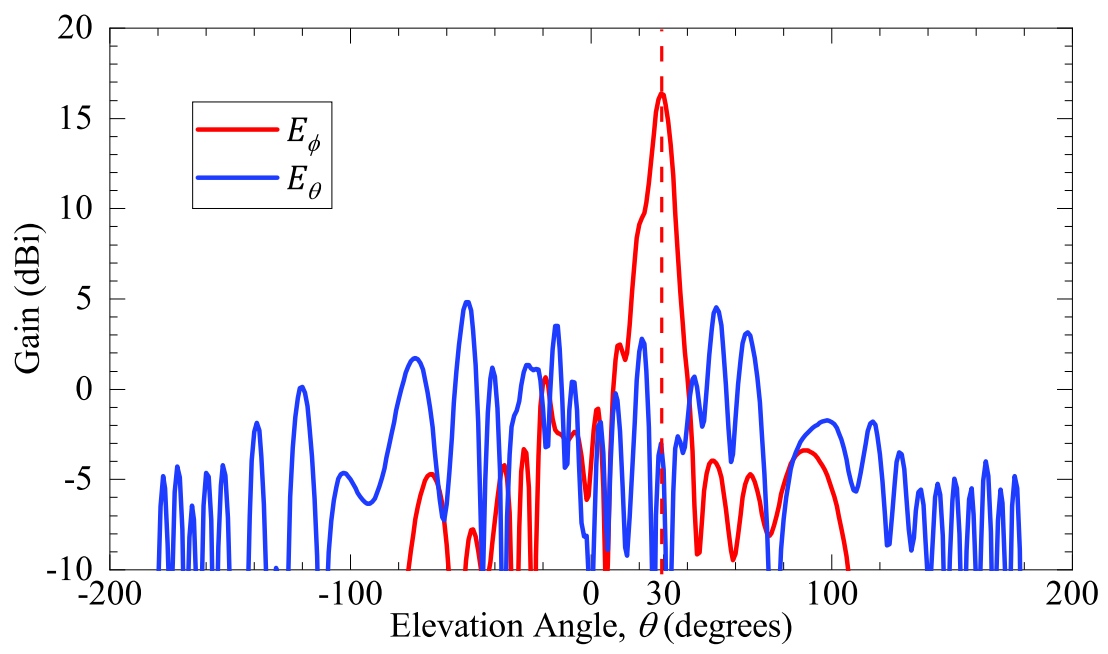

(b)

Figure 13. Cont. 


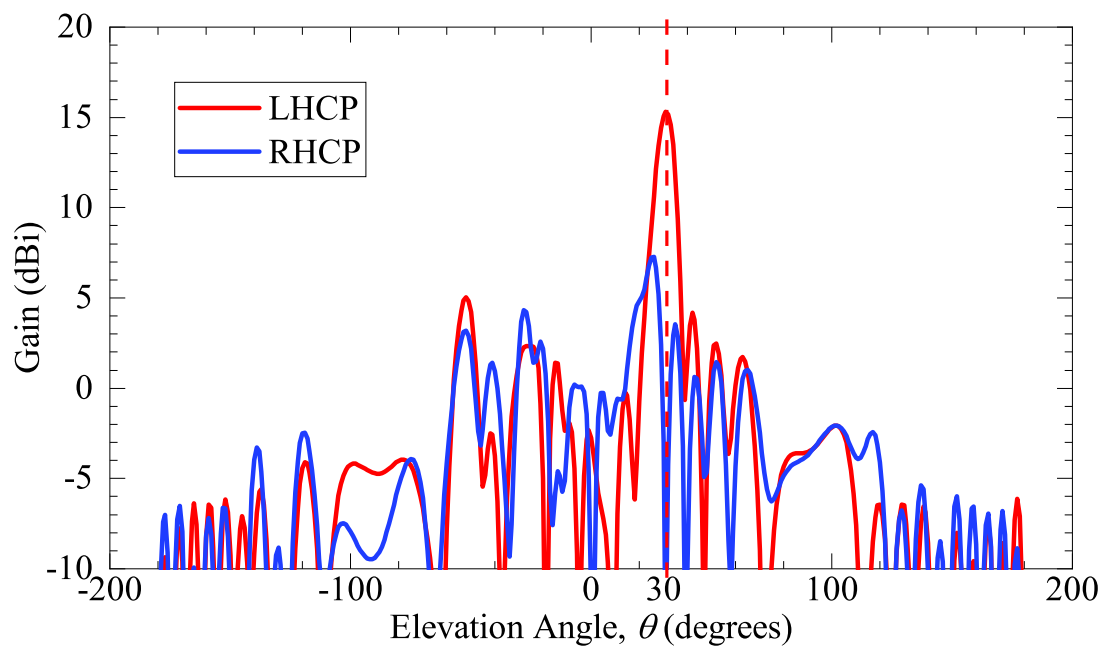

(c)

Figure 13. The E-plane radiation patterns obtained at $12 \mathrm{GHz}$ when the antenna is excited by the (a) first, (b) second, and (c) both sources. $12 \mathrm{GHz}$. The metasurface is realized using Rogers RT/Duroid 5880 substrate of thickness $3.175 \mathrm{~mm}$ and a dielectric constant of 2.2. The average surface reactance and the modulation index are 255 and 31 ohms, respectively [23].

The metasurface forms a pencil beam in the desired direction with a vertical, horizontal, and circular polarization when excited by the first, second, and both sources, respectively.

The sense of rotation of the circular polarization depends on the $90^{\circ}$ phase lead or lag between the two linear polarizations. A circularly-polarized beam with the other sense of rotation can be formed by externally including a $180^{\circ}$ phase shift between the two feeds or by including another source and modulating the metasurface with the superimposition of the surface reactance pertaining to the three sources [6].

Employing metasurfaces and the principle of holography has resulted in a simple configuration of a multi-polarization high-gain LWA, which would otherwise be difficult to achieve. While the presence of multiple sources, yields the metasurface polarization diverse, internal reconfiguration of polarization can be potentially achieved by including switches between the patches. The difference between the surface-reactance profiles that lead to horizontal and vertical polarizations, is the inclusion of a $180^{\circ}$ phase difference on either side of the major or minor axis of the ellipses. Electronic switches like PIN diodes can be used to include the $180^{\circ}$ phase shift, thus resulting in internal reconfiguration; this yields to a metasurface, which is polarization reconfigurable. While having a switch in every unit cell would be expensive and cumbersome to process, the number of switches can be optimized by a judicious design.

\section{A Van Atta Retrodirective Microstrip Antenna Array with Low Backscattering}

While the previous two sections demonstrate the application of metasurfaces for surface-wave antenna arrays and leaky-wave antennas, this section focuses on the reconfiguration and improvement of van Atta arrays using AMC-based metasurfaces. In a van Atta reflectarray, the fields reradiated by the antennas are necessary while the fields scattered from the structure (i.e., structural mode) are undesired. Hence, metasurfaces are employed to eliminate these scattered fields from the structural mode of the van Atta retrodirective reflectarray.

\subsection{Theory of Passive Retrodirective Arrays}

A schematic diagram of a 1-D van Atta array is illustrated in Figure 14, where four array elements are connected using the two transmission lines (i.e., elements ' 1 ' and ' 4 ' are connected with each-other while element ' 2 ' is connected to element ' 3 '). In order to direct the reradiated waves 
back to the direction of the incident wave, these transmission lines must either be of equal lengths or the difference between their lengths should be a multiple of the wavelength. Since these two transmission lines transfer the signals received by elements ' 1 ' and ' 2 ' to elements ' 3 ' and ' 4 ', respectively; the lengths of the transmission lines connecting the antenna element should be kept equal to make the functioning frequency independent. Therefore both transmission lines have equal lengths, $l_{1}=l_{2}=l$. When a plane wave is incident obliquely upon the array at an angle $\theta_{i}$, a phase delay $\Delta$ between the antenna elements is introduced [44]

$$
\Delta=k_{0} d \sin \theta_{t}
$$

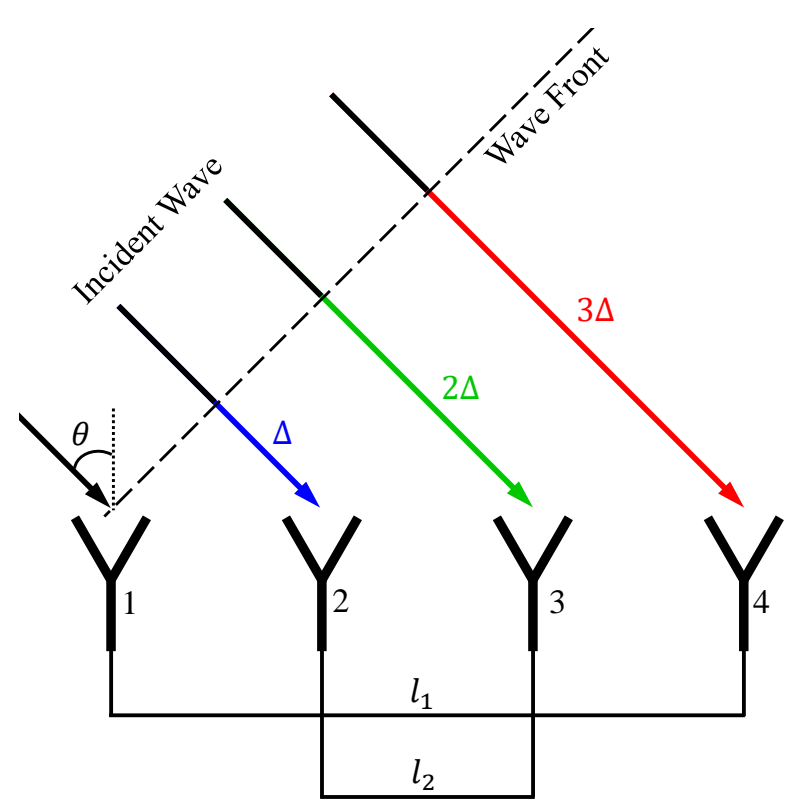

Figure 14. A schematic diagram of a 1-D van Atta retrodirective array where four elements are used in the array.

The monostatic RCS of the retrodirective reflector, based on the reradiated fields only, can be derived following the approach in $[38,39]$ and represented by

$$
\sigma^{R}=\frac{\lambda_{0}^{2}}{4 \pi} G_{0}^{2}
$$

where $G_{0}$ is the gain of the array in the principle plane, $\theta$ is the incident/elevation angle, and $\lambda_{0}$ is the free-space wavelength. The monostatic RCS $\left(\sigma^{R}\right)$ of Equation (5) is only based on the fields reradiated $\left(\mathbf{E}^{\mathbf{R}}\right)$ by the reflector array. However, the total RCS by the reflector array $\left(\sigma^{T}\right)$ corresponds to the total radiated fields $\left(\mathbf{E}^{\mathbf{T}}\right)$ which can be determined by adding the reradiated fields $\left(\mathbf{E}^{\mathbf{R}}\right)$ and the scattered fields by the structure $\left(\mathrm{E}^{\mathrm{S}}\right)$. Figure 15 shows a comparison of the ideal monostatic RCS between a retrodirective reflector $\left(\sigma^{R}\right)$ of Equation (5) and a flat perfect electric conductor (PEC) plate, where both have the same size.

The retrodirective response is obtained by having a planar array of $4 \times 4$ radiating elements, each of gain $\left[G_{0}(\theta)\right]$ and with $0.5 \lambda_{0}$ spacing between them. Thus, the total size of both the reflector array and the flat PEC plate is $2 \lambda_{0} \times 2 \lambda_{0}$. It is clear from Figure 15 that the fully passive reflector array can offer a maximum reradiation towards the direction of incidence. However, due to the limitation of the patch antenna towards the grazing angles, the maximum reradiation pattern follows the radiation pattern of the single antenna. 


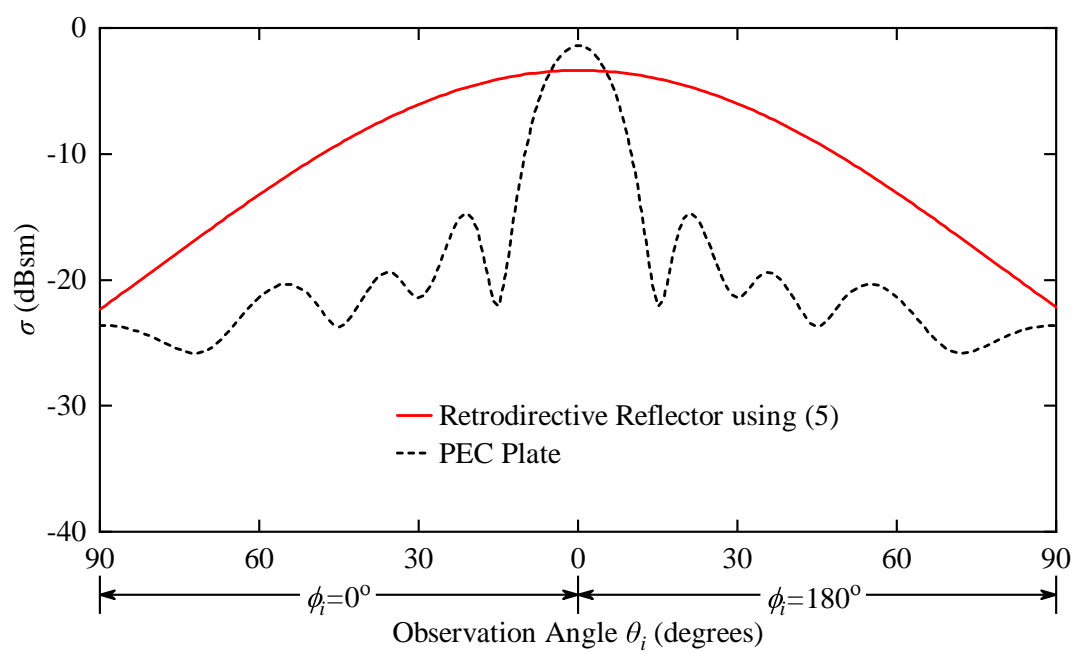

Figure 15. Comparison of the ideal monostatic RCS between a retrodirective reflector $\left(\sigma^{R}\right)$ obtained by using (5) and a flat PEC.

\subsection{Design of the Patch Antenna and Its Feeding Network}

In principle, a retrodirective array can be implemented with any kind of antenna element where the bandwidth performance and beamwidth of the array will be directly impacted by the performance parameters of the single radiating element. Due to their design simplicity and their low profile, patch antennas are chosen to design the utilized retrodirective array.

\subsubsection{Aperture-Coupled Patch Antenna with Microstrip Line Feeding Network}

Figure 16 illustrates the retrodirective reflector that is constructed using a $4 \times 4$ finite array of rectangular microstrip-patch antennas.

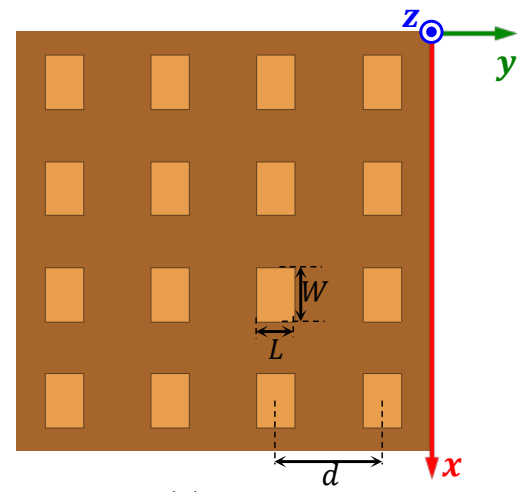

(a)

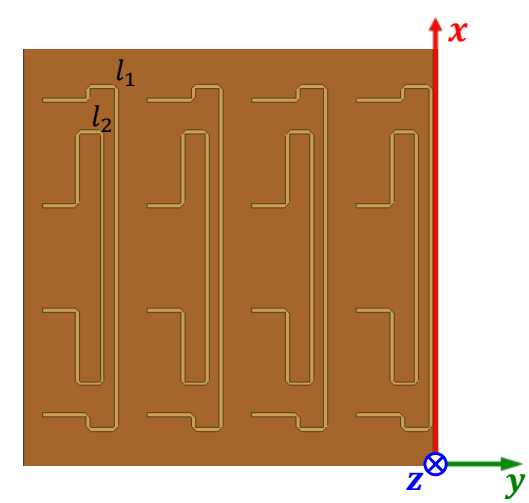

(b)

Figure 16. The geometry of the four by four finite array of rectangular microstrip patch antennas that are connected in the van Atta configuration in the $x z$-plane, (a) top. (b) bottom.

The elements are interconnected as per the van Atta configuration (i.e., illustrated in Figure 14) in the $x z$-plane. The feeding network is constructed using a $50 \mathrm{ohms} \mathrm{microstrip} \mathrm{transmission} \mathrm{lines} \mathrm{of}$ equal length (i.e., $l=l_{1}=l_{2}$ ). The dimensions of the square array along the $x$ - and $y$-direction are $120 \mathrm{~mm}$, corresponding to $2 \lambda_{0} ; \lambda_{0}$ is the free-space wavelength at $5 \mathrm{GHz}$. The top side of the structure consists of 16 patch elements resonating at $5 \mathrm{GHz}$. With reference to Figure 16, the array spacing is $d=30 \mathrm{~mm}\left(0.5 \lambda_{0}\right)$, and the patch dimensions are $L=11 \mathrm{~mm}$ and $W=15.4 \mathrm{~mm}$. All the elements are designed on Rogers-RO3006 dielectric substrate (thickness $=1.28 \mathrm{~mm}, \varepsilon_{r}=6.15$ and $\tan \delta=0.0022$ at $10 \mathrm{GHz}$ ) backed by a PEC. 
The above-mentioned design was (modeled in HFSS) illuminated by a plane wave incident along the principal plane ( $x z$-plane) defined by $\phi_{i}=0^{\circ}, 180^{\circ}$ and for $0^{\circ} \leq \theta_{i} \leq 90^{\circ}$. The configuration was simulated with two different lengths $(l)$ of the transmission lines that are connecting the antenna elements. The total monostatic RCSs $\left(\sigma^{T}\right)$ of the retrodirective array for the two transmission lines are depicted in Figure 17. The difference between the two responses is primarily due to the change in the phase of $\mathbf{E}^{\mathbf{R}}$ with respect to $\mathbf{E}^{\mathbf{S}}$ when the length of the transmission lines is changed.

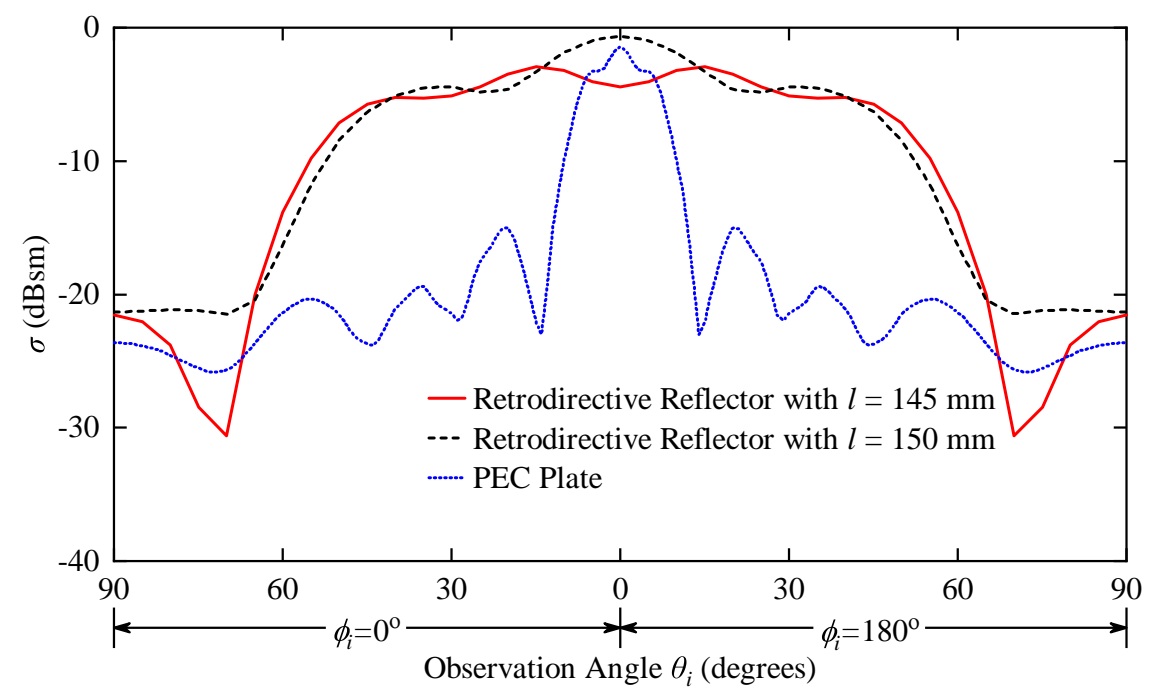

Figure 17. The monostatic RCSs of a retrodirective array with two different transmission line lengths compared to the monostatic RCS of a PEC plate of the same size.

Table 1 summarizes the values of the broadside RCS $\left(\theta_{i}=0^{\circ}\right)$ of the retrodirective array for the two different lengths of transmission line. It is assumed that the phase of the scattered fields by the array structure $\left(\mathbf{E}^{\mathbf{S}}\right)$ does not vary significantly with the change of length $l$ of the transmission line (this will be verified in the next section). Thus, the total scattered $\left(\mathbf{E}^{\mathbf{T}}\right)$ will depend on the varying phase introduced by the transmission line. As seen in Table 1, for the first length of $l=145 \mathrm{~mm}$, the two components $\left(\mathbf{E}^{\mathbf{S}}\right.$ and $\left.\mathbf{E}^{\mathbf{R}}\right)$ of $\mathbf{E}^{\mathbf{T}}$ added destructively near broadside incidence, while for the second length of $l=150 \mathrm{~mm}$, the two components are in-phase and thus the total RCS is higher than the reradiated $\sigma^{R}$ obtained using Equation (5). With the current retrodirective setup (aperture-coupled patch antenna with microstrip line feeding network), it is extremely difficult to extract two components of the total scattered fields. In the next section, it will be shown that the virtual feeding network can be used to identify the two separate components of the total scattered field, and it can permit the targeting and cancellation of one of the components, especially the scattering by the structural mode, which is undesirable.

Table 1. The broadside $\left(\theta_{i}=0^{\circ}\right)$ RCS of the retrodirective array for two different coaxial cable lengths.

\begin{tabular}{cccc}
\hline & $l=\mathbf{1 4 5} \mathbf{~ m m}$ & $l=\mathbf{1 5 0} \mathbf{~ m m}$ & Analytical Using (5) \\
\hline$\sigma(\mathrm{dBsm})$ & -4.42 & -0.63 & -3.24 \\
\hline
\end{tabular}

\subsubsection{Aperture-Coupled Patch Antenna with Virtual Feeding Network}

An alternative method for simulating the retrodirective reflector explained in Section 4.2.1 is to use a virtual feeding network. The basic concept of a virtual feeding network is to capture how much voltage (magnitude and phase) is received by each antenna element, and then feed the measured voltages to the respective antenna elements following the van Atta configuration shown in Figure 14. All the radiating elements of the array are terminated with an impedance equal to the input impedance of the antenna (ideally $50 \mathrm{ohms}$ ). Then, at the termination point, the received voltage 
from the illuminating plane wave is determined by integrating along the electric field lines around the microstrip lines, similar to quasi-static simulators. This integration is performed based on the mode of propagation, which is Quasi-Transverse Electromagnetic (Quasi-TEM) as illustrated in Figure 18 [45]. The above-mentioned process is achieved by using a co-simulation between the full-wave simulator (Ansys Electromagnetic Suite/HFSS), and a Python code that is written to integrate the electric fields and then feed those calculated voltages again to their respective elements in the full-wave simulator. Figure 19 shows the structure that was configured in HFSS. Here, each of the antenna ports (i.e., transmitting antennas) is terminated with an impedance that is equal to the input impedance of the corresponding connected antenna (i.e., receiving antenna) to replicate the connection between them.

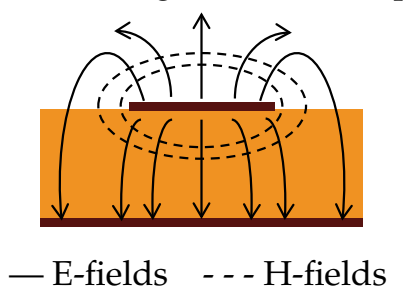

Figure 18. Electric and magnetic field lines for the TEM mode of a coaxial line.

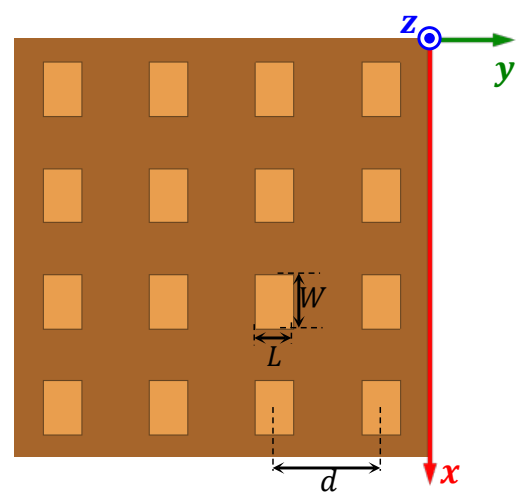

(a)

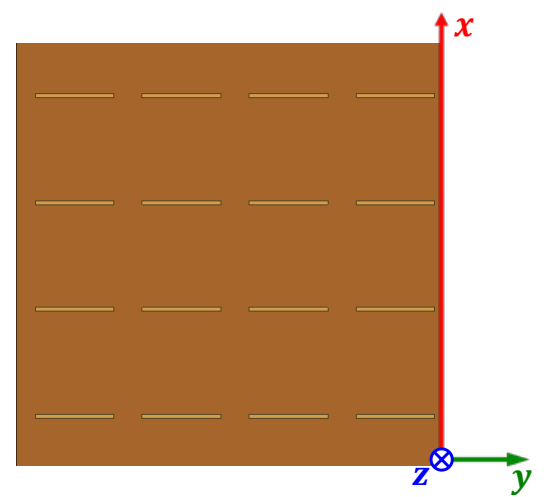

(b)

Figure 19. (a) Top and (b) bottom geometries of the $4 \times 4$ finite array of rectangular microstrip-patch antennas that are terminated and virtually connected in the van Atta configuration in the $x z$-plane.

Similar to the arrangement of Section 4.2.1, the structure is illuminated by a plane wave incident in the principal plane ( $x z$-plane) defined by $\phi_{i}=0^{\circ}, 180^{\circ}$ and for $0 \leq \theta_{i} \leq 90^{\circ}$. Again, by using the Python script, the voltage captured by each antenna element at each incident angle is recorded and fed again to their counterpart reradiating element. This process allows for the decoupling of the total scattered fields $\mathbf{E}^{\mathbf{T}}$ into the two main components: reradiated fields $\mathbf{E}^{\mathbf{R}}$ and structural mode scattering $\mathbf{E}^{\mathrm{S}}$; their corresponding RCSs are shown in Figure 20. It can be shown that the phase of $\mathrm{E}^{\mathrm{S}}$ is primarily dependent on the structure, and it is not impacted by the phase introduced by the connecting cables. However, the phase of $\mathbf{E}^{\mathbf{R}}$ can be altered based on the connecting cables. Thus, to illustrate the impact of the phase difference $\left(\Delta_{R S}\right)$ at the broadside $\left(\theta_{i}\right)$ between $\mathbf{E}^{\mathbf{S}}$ and $\mathbf{E}^{\mathbf{R}}$, the total RCS by the retrodirective array is shown in Figure 20 when $\Delta_{R S}=0^{\circ}$ and $180^{\circ}$, respectively. 


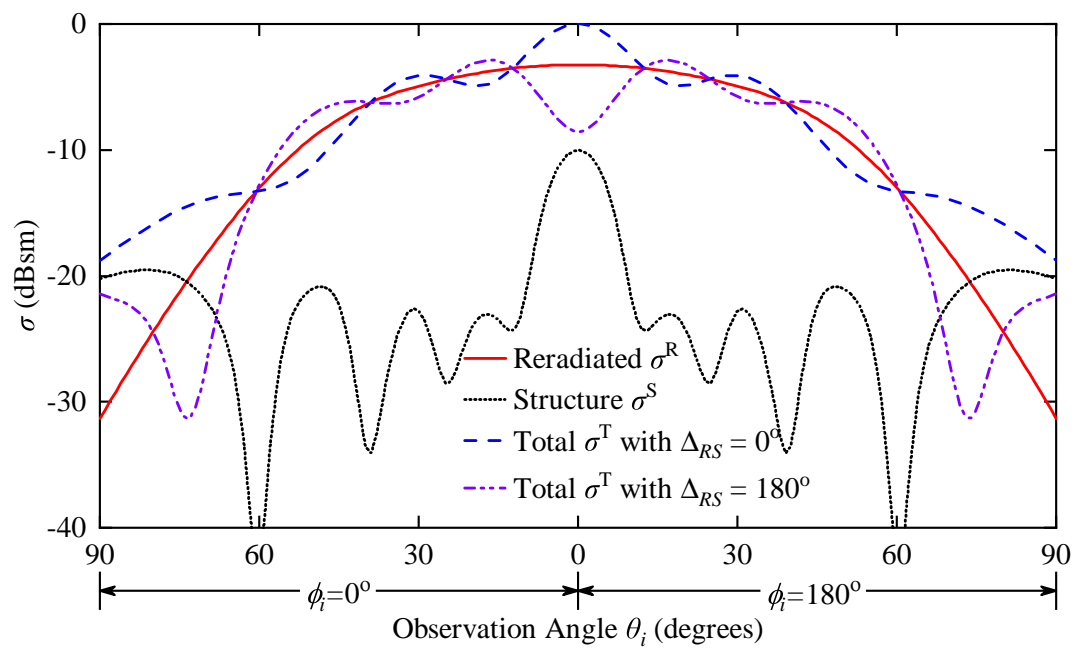

Figure 20. The monostatic RCSs based on the reradiated, scattered, and the total fields when the phase difference $\left(\Delta_{R S}\right)$ between $\mathbf{E}^{\mathbf{R}}$ and $\mathbf{E}^{\mathbf{S}}$ is $0^{\circ}$ and $180^{\circ}$.

\subsection{Retrodirective Reflector with Low Backscattering Using Metamaterials}

It was shown in the previous section that the scattered fields from the structure introduce ripples in the total RCS by the reflector, especially near the broadside, where the RCS of the structure is large. Ideally, $\mathbf{E}^{\mathbf{S}}$ can be canceled by a judiciously synthesized metasurface, like that of the widely-known concept of checkerboard metasurfaces [46].

\section{Design of Metasurfaces Based on AMCs for RCS Reduction of Retrodirective Array}

The RCS of the array structure can be reduced by placing an AMC, whose reflection is similar in magnitude but with a $180^{\circ}$ phase difference compared to that of $\mathbf{E}^{\mathbf{S}}$. As shown in Figure 20, the RCS of the structural mode is around $-10 \mathrm{dBsm}$ and the phase of its reflected fields is around $130^{\circ}$. Thus, an AMC surface of square patches is designed where their width and unit cell dimensions are selected to have a reflection phase of around $-50^{\circ}$ and an RCS of $-10 \mathrm{dBsm}$, similar to the monostatic RCS corresponding to the structural mode of the antenna.

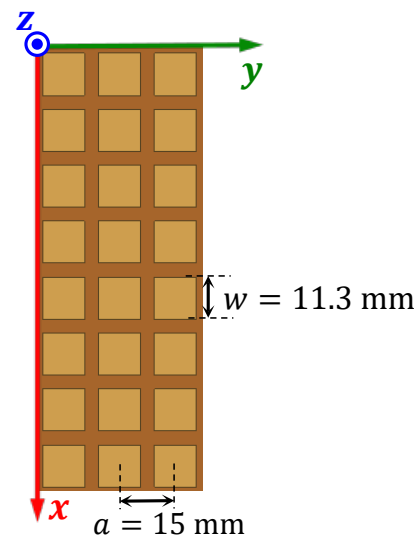

(a)

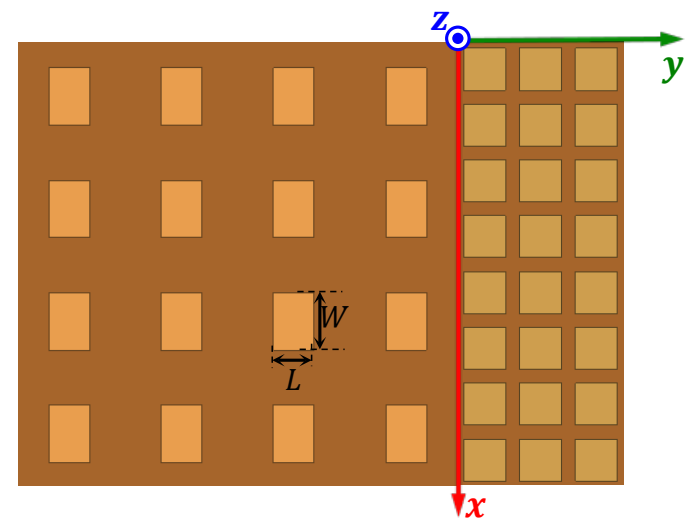

(b)

Figure 21. Geometries of (a) the finite array of the AMC and (b) the $4 \times 4$ array of rectangular microstrip patch antennas combined with the finite AMC array for canceling the RCS of the structure.

The geometries of the synthesized finite sized AMC metasurface are depicted in Figure 21a. As a result, as illustrated in Figure 21b, the final design of the proposed retrodirective reflector is obtained by placing the original antenna array of Figure 19 adjacent to the synthesized AMC of 
Figure 21a. This configuration will introduce fields by the AMC with a phase difference of $180^{\circ}$ compared to the phase of the scattered fields by the antenna structure.

This design is then illuminated by following a similar arrangement to the retrodirective reflector without the AMC, as explained previously in Section 4.2. The monostatic RCSs due to the antenna structure, reradiated fields, and the total fields are shown in Figure 22. It can be seen that regardless of the phase introduced by the connecting cables that impact the phase of the reradiated fields only, the monostatic RCS caused by the structure $\sigma^{S}$ is reduced at and near broadside. This causes the total RCS to have smaller ripples and the performance becomes less sensitive to the interference by the scattered fields of the structure.

The RCS, which corresponds to the fields scattered by the antenna structure, can be reduced by placing a metasurface with a similar RCS and $180^{\circ}$ phase difference with respect to the reflected fields of the antenna array. The range of the phase difference can be relaxed to achieve an RCS reduction of at least $10 \mathrm{~dB}$ when the phase difference is maintained within $(180 \pm 37)^{\circ}$ [46,47]. Using this criterion, the RCS of various antennas has been reduced for more than $10 \mathrm{~dB}$ over a wide frequency band $[46,48,49]$; furthermore, a 10-dB RCS reduction is not necessary here. It is sufficient that the RCS of the scattered fields is lower than that of the reradiated fields by a certain factor based on the acceptable level of the ripples in the total field. Consequently, the phase-difference criterion of $(180 \pm 37)^{\circ}$ can be relaxed further, and a much broader operational bandwidth can be attained. In this model, the metasurface is designed to achieve the RCS-reduction at the operating frequency of the van Atta reflector to validate the concept, which can be applied over a broader frequency range.

The scanning capability of the van Atta reflector depends on the overall gain of the array $\left(G_{0}\right)$ as given by Equation (5), where $G_{0}$ is determined by the radiation pattern of the single element and the spacing between them. Thus, individual radiating elements with broad beamwidths result in a reflector that responds to larger incident angles. However, antennas with broad beamwidths usually have a lower gain. As a figure of merit, the 10-dB beamwidth of the array is considered as its scanning range. In this design, the monostatic RCS plotted in Figure 22 shows that the 10- $\mathrm{dB}$ beamwidth of the designed array is about $120^{\circ}$ in the principle scanning plane (i.e., $x z$-plane).

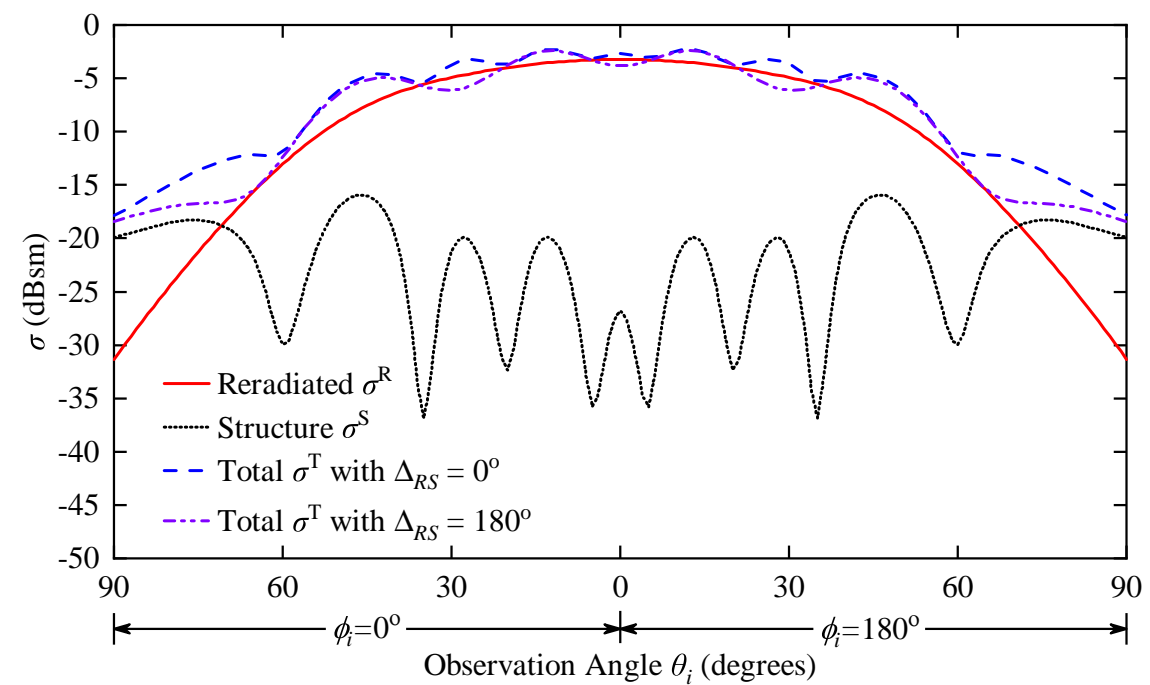

Figure 22. The monostatic RCSs based on the reradiated, scattered, and the total fields when the phase difference $\left(\Delta_{R S}\right)$ between $\mathbf{E}^{\mathbf{R}}$ and $\mathbf{E}^{\mathbf{S}}$ is $0^{\circ}$ and $180^{\circ}$.

\section{Conclusions}

The application of metasurfaces in the reconfiguration and enhancement of the performance of surface-wave antenna arrays, holographic leaky-wave antennas, and retrodirective arrays is illustrated. Square ring-element arrays embedded within metasurfaces are proposed for linear and circular polarizations. The coupling between the ports and their effect on the broadside radiation are discussed 
and highlighted. For practicality, parallel feed networks are incorporated with the two proposed designs. With the incorporation of metasurfaces, a high realized gain and a high aperture efficiency, compared to a conventional square ring array, are observed. A prototype was fabricated and a good agreement between the simulations and measurements was demonstrated.

Metasurface LWAs designed using the holographic principle are reviewed. In particular, polarization-diverse holographic metasurfaces that can form a pencil beam with a horizontal, vertical, or circular polarization are discussed. A potential technique to incorporate electronic reconfigurability is proposed.

As the third area of illustration, metasurfaces are employed to improve the monostatic RCS performance of retrodirective arrays. A co-simulation method that allows for decoupling the total scattered fields is introduced. Such a simulation allows the identification and cancellation of the undesired component in the scattered fields (those scattered by the antenna structure). It is shown that this work enables the use of retrodirective arrays comprised of simple patch antennas while simultaneously addressing the issue of backscattering by using metasurfaces based on AMCs.

Author Contributions: Conceptualization, S.R. and C.A.B.; methodology, M.A., M.A.A. and S.R.; software, M.A., M.A.A., and S.R.; validation, C.R.B., C.A.B. and A.Y.M.; formal analysis, S.R., A.Y.M. and C.A.B.; investigation, M.A., M.A.A. and S.R.; resources, M.A., M.A.A. and S.R.; data curation, M.A., M.A.A., S.R. and C.R.B.; writing—original draft preparation, M.A., M.A.A. and S.R.; writing—review and editing, C.A.B., S.R. and A.Y.M.; visualization, M.A., M.A.A. and S.R. All authors have read and agreed to the published version of the manuscript.

Funding: This research received no external funding.

Conflicts of Interest: The authors declare no conflict of interest.

\section{References}

1. Bukhari, S.S.; Vardaxoglou, J.; Whittow, W. A Metasurfaces Review: Definitions and Applications. Appl. Sci. 2019, 9, 2727. [CrossRef]

2. Pfeiffer, C.; Grbic, A. Metamaterial Huygens surfaces: Tailoring wave fronts with reflectionless sheets. Phys. Rev. Lett 2013, 110, 197401. [CrossRef] [PubMed]

3. Maci, S.; Minatti, G.; Casaletti, M.; Bosiljevac, M. Metasurfing: Addressing Waves on Impenetrable Metasurfaces. IEEE Antennas Wirel. Propag. Lett. 2011, 10, 1499-1502. [CrossRef]

4. Fong, B.H.; Colburn, J.S.; Ottusch, J.J.; Visher, J.L.; Sievenpiper, D.F. Scalar and tensor holographic artificial impedance surfaces. IEEE Trans. Antennas Propag. 2010, 58, 3212-3221. [CrossRef]

5. Ramalingam, S.; Balanis, C.A.; Birtcher, C.R.; Pandi, S.; Shaman, H.N. Axially Modulated Cylindrical Metasurface Leaky-Wave Antennas. IEEE Antennas Wirel. Propag. Lett. 2018, 17, 130-133. [CrossRef]

6. Ramalingam, S.; Balanis, C.A.; Birtcher, C.R.; Pandi, S.; Shaman, H.N. Polarization-diverse holographic metasurfaces. IEEE Antennas Wirel. Propag. Lett. 2019, 18, 264-268. [CrossRef]

7. Modi, A.Y.; Balanis, C.A.; Birtcher, C.R.; Shaman, H.N. Novel Design of Ultrabroadband Radar Cross Section Reduction Surfaces Using Artificial Magnetic Conductors. IEEE Trans. Antennas Propag. 2017, 65, 5406-5417. [CrossRef]

8. Modi, A.Y.; Alyahya, M.A.; Balanis, C.A.; Birtcher, C.R. Metasurface-Based Method for Broadband RCS Reduction of Dihedral Corner Reflectors With Multiple Bounces. IEEE Trans. Antennas Propag. 2020, 68, 1436-1447. [CrossRef]

9. Ramalingam, S.; Balanis, C.A.; Birtcher, C.R.; Pandi, S. Analysis and Design of Checkerboard Leaky-Wave Antennas With Low Radar Cross Section. IEEE Open J. Antennas Propag. 2020, 1, 26-40. [CrossRef]

10. Alharbi, M.S.; Balanis, C.A.; Birtcher, C.R. Hybrid Circular Ground Planes for High-Realized-Gain Low-Profile Loop Antennas. IEEE Antennas Wirel. Propag. Lett. 2018, 17, 1426-1429. [CrossRef]

11. Alharbi, M.S.; Balanis, C.A.; Birtcher, C.R. Performance Enhancement of Square-Ring Antennas Exploiting Surface-Wave Metasurfaces. IEEE Antennas Wirel. Propag. Lett. 2019, 18, 1991-1995. [CrossRef]

12. Costa, F.; Luukkonen, O.; Simovski, C.R.; Monorchio, A.; Tretyakov, S.A.; de Maagt, P.M. TE Surface Wave Resonances on High-Impedance Surface Based Antennas: Analysis and Modeling. IEEE Trans. Antennas Propag. 2011, 59, 3588-3596. [CrossRef] 
13. Yang, F.; Aminian, A.; Rahmat-Samii, Y. A Novel Surface-Wave Antenna Design Using a Thin Periodically Loaded Ground Plane. Microw. Opt. Technol. Lett. 2005, 47, 240-245. [CrossRef]

14. Yang, F.; Rahmat-Samii, Y.; Kishk, A. Low-Profile Patch-Fed Surface Wave Antenna With a Monopole-Like Radiation Pattern. IET Microw. Antennas Propag. 2007, 1, 261-266. [CrossRef]

15. Feng, G.; Chen, L.; Xue, X.; Shi, X. Broadband Surface-Wave Antenna With a Novel Nonuniform Tapered Metasurface. IEEE Antennas Wirel. Propag. Lett. 2017, 16, 2902-2905. [CrossRef]

16. Lin, F.H.; Chen, Z.N. Low-Profile Wideband Metasurface Antennas Using Characteristic Mode Analysis. IEEE Trans. Antennas Propag. 2017, 65, 1706-1713. [CrossRef]

17. Yang, W.; Chen, S.; Che, W.; Xue, Q.; Meng, Q. Compact High-Gain Metasurface Antenna Arrays Based on Higher-Mode SIW Cavities. IEEE Trans. Antennas Propag. 2018, 66, 4918-4923. [CrossRef]

18. Zhu, H.L.; Cheung, S.W.; Chung, K.L.; Yuk, T.I. Linear-to-Circular Polarization Conversion Using Metasurface. IEEE Trans. Antennas Propag. 2013, 61, 4615-4623. [CrossRef]

19. Ta, S.X.; Park, I. Low-Profile Broadband Circularly Polarized Patch Antenna Using Metasurface. IEEE Trans. Antennas Propag. 2015, 63, 5929-5934. [CrossRef]

20. Wu, Z.; Li, L.; Li, Y.; Chen, X. Metasurface Superstrate Antenna With Wideband Circular Polarization for Satellite Communication Application. IEEE Antennas Wirel. Propag. Lett. 2016, 15, 374-377. [CrossRef]

21. Ta, S.X.; Park, I. Compact Wideband Circularly Polarized Patch Antenna Array Using Metasurface. IEEE Antennas Wirel. Propag. Lett. 2017, 16, 1932-1936. [CrossRef]

22. Patel, A.M.; Grbic, A. A printed leaky-wave antenna based on a sinusoidally-modulated reactance surface. IEEE Trans. Antennas Propag. 2011, 59, 2087-2096. [CrossRef]

23. Ramalingam, S. Impedance Modulated Metasurface Antennas. Ph.D. Dissertation, Arizona State University, Tempe, AZ, USA, 2020.

24. Minatti, G.; Caminita, F.; Casaletti, M.; Maci, S. Spiral leaky-wave antennas based on modulated surface impedance. IEEE Trans. Antennas Propag. 2011, 59, 4436-4444. [CrossRef]

25. Pandi, S.; Balanis, C.A.; Birtcher, C.R. Design of Scalar Impedance Holographic Metasurfaces for Antenna Beam Formation with Desired Polarization. IEEE Trans. Antennas Propag. 2015, 63, 3016-3024. [CrossRef]

26. Minatti, G.; Caminita, F.; Martini, E.; Sabbadini, M.; Maci, S. Synthesis of modulated-metasurface antennas with amplitude, phase, and polarization control. IEEE Trans. Antennas Propag. 2016, 64, 3907-3919. [CrossRef]

27. Bodehou, M.; Martini, E.; Maci, S.; Huynen, I.; Craeye, C. Multibeam and Beam Scanning With Modulated Metasurfaces. IEEE Trans. Antennas Propag. 2020, 68, 1273-1281. [CrossRef]

28. Li, M.; Tang, M.; Xiao, S. Design of a LP, RHCP and LHCP Polarization-Reconfigurable Holographic Antenna. IEEE Access 2019, 7, 82776-82784. [CrossRef]

29. Van Atta, L.C. Electromagnetic Reflector. U.S. Patent 2,908,002, 6 October 1959.

30. Sharp, E.; Diab, M. Van atta reflector array. IEEE Trans. Antennas Propag. 1960, 8, 436-438. [CrossRef]

31. Appel-Hansen, J. A van atta reflector consisting of half-wave dipoles. IEEE Trans. Antennas Propag. 1996, 14, 694-700. [CrossRef]

32. Chung, S.J.; Chang. K. A retrodirective microstrip antenna array. IEEE Trans. Antennas Propag. 1998, 46, 1802-1809. [CrossRef]

33. Li, Y.; Jandhyala, V. Design of retrodirective antenna arrays for short-range wireless power transmission. IEEE Trans. Antennas Propag. 2012, 60, 206-211. [CrossRef]

34. Wang, X.; Sha, S.; He, J.; Guo, L.; Lu, M. Wireless power delivery to low-power mobile devices based on retro-reflective beamforming. IEEE Antennas Wirel. Propag. Lett. 2014, 13, 919-922. [CrossRef]

35. Ettorre, M.; Alomar, W.A.; Grbic, A. Radiative wireless power-transfer system using wideband, wide-angle slot arrays. IEEE Trans. Antennas Propag. 2017, 65, 2975-2982. [CrossRef]

36. Tseng, W.J.; Chung, S.B.; Chang, K. A planar van atta array reflector with retrodirectivity in both e-plane and h-plane. IEEE Trans. Antennas Propag. 2000, 48, 173-175. [CrossRef]

37. Hester, J.G.D.; Tentzeris, M.M. Inkjet-printed flexible mm-wave van-atta reflectarrays: A solution for ultralong-range dense multitag and multisensing chipless rfid implementations for iot smart skins. IEEE Trans. Microw. Theory Tech. 2016, 64, 4763-4773. [CrossRef]

38. Christie, S.; Cahill, R.; Buchanan, N.B.; Fusco, V.F.; Mitchell, N.; Munro, Y.V.; Maxwell-Cox, G. Rotman lens-based retrodirective array. IEEE Trans. Antennas Propag. 2012, 60, 1343-1351. [CrossRef]

39. Ettorre, M.; Alomar, W.A.; Grbic, A. 2-d van atta array of wideband, wideangle slots for radiative wireless power transfer systems IEEE Trans. Antennas Propag. 2018, 66, 4577-4585. [CrossRef] 
40. Sievenpiper, D.F. High-Impedance Electromagnetic Surfaces. Ph.D. Dissertation, University of California, Los Angeles, CA, USA, 1999.

41. Yang, F.; Rahmat-Samii, Y. Microstrip antennas integrated with electromagnetic band-gap (EBG) structures: A Low mutual coupling design for array applications. IEEE Trans. Antennas Propag. 2003, 51, 2936-2946. [CrossRef]

42. Quarfoth, R. Anisotropic Artificial Impedance Surfaces. Ph.D. Dissertation, University of California, San Diego, CA, USA, 2014.

43. Balanis, C.A. Advanced Engineering Electromagnetics, 2nd ed.; Wiley: Hoboken, NJ, USA, 2012.

44. Balanis, C.A. Antenna Theory: Analysis and Design, 4th ed.; John Wiley \& Sons: Hoboken, NJ, USA, 2016.

45. Pozar, D.M. Microwave Engineering, 4th ed.; John Wiley \& Sons: Hoboken, NJ, USA, 2011.

46. Modi, A.Y.; Balanis, C.A.; Birtcher, C.R.; Shaman, H.N. New Class of RCS-Reduction Metasurfaces Based on Scattering Cancellation Using Array Theory. IEEE Trans. Antennas Propag. 2019, 67, 298-308. [CrossRef]

47. Chen, W.; Balanis, C.A.; Birtcher, C.R. Checkerboard EBG surfaces for wideband radar cross section reduction. IEEE Trans. Antennas Propag. 2015, 63, 2636-2645. [CrossRef]

48. Liu, Y.; Li, K.; Jia, Y.; Hao, Y.; Gong, S.; Guo, Y.J. Wideband RCS Reduction of a Slot Array Antenna Using Polarization Conversion Metasurfaces. IEEE Trans. Antennas Propag. 2016, 64, 326-331. [CrossRef]

49. Zheng, Y.; Gao, J.; Zhou, Y.; Ziangyu, C.; Yang, H.; Li, S.; Li, T. Wideband Gain Enhancement and RCS Reduction of Fabry-Perot Resonator Antenna With Chessboard Arranged Metamaterial Superstrate. IEEE Trans. Antennas Propag. 2018, 67, 590-599. [CrossRef]

(C) 2020 by the authors. Licensee MDPI, Basel, Switzerland. This article is an open access article distributed under the terms and conditions of the Creative Commons Attribution (CC BY) license (http://creativecommons.org/licenses/by/4.0/). 